\title{
Sedimentation Velocity and Potential in a Dilute Suspension of Charged Composite Spheres
}

\author{
Huan J. Keh ${ }^{1}$ and Yung C. Liu \\ Department of Chemical Engineering, National Taiwan University, Taipei 106-17, Taiwan, Republic of China
}

Received May 27, 1997; accepted August 19, 1997

The sedimentation of a charged composite particle composed of a solid core and a surrounding porous shell in an electrolyte solution is analytically studied. In the solvent-permeable and ionpenetrable porous surface layer of the particle, idealized hydrodynamic frictional segments with fixed charges are assumed to distribute at a uniform density. The equations which govern the ionic concentration distributions, the electric potential profile, and the fluid flow field inside and outside the surface layer of a charged composite particle migrating in an unbounded solution are linearized assuming that the system is only slightly distorted from equilibrium. Using a perturbation method, these linearized equations are solved for a composite sphere with the charge densities of the rigid core surface and of the surface layer as the small perturbation parameters. An analytical expression for the settling velocity of the composite sphere in closed form is obtained from a balance among its gravitational, electrostatic, and hydrodynamic forces. The result demonstrates that the presence of the fixed charges in the composite sphere slows down its settling velocity relative to that of an uncharged one. A closed-form formula for the sedimentation potential in a dilute suspension of identical charged composite spheres is also derived by using the requirement of zero net electric current. The Onsager reciprocal relation is found to be satisfied between sedimentation and electrophoresis. It is shown that spherically-symmetric "neutral" composite particles ( bearing no net charge) can undergo electrophoresis, induce sedimentation potential, and experience a smaller settling velocity relative to corresponding uncharged particles. The direction of the electrophoretic velocity or the induced potential gradient is determined by the fixed charges in the porous surface layers of the particles. In the limiting cases, the analytical solutions describing the sedimentation velocity and sedimentation potential ( or electrophoretic mobility) for charged composite spheres reduce to those for charged solid spheres and for charged porous spheres. (1) 1997 Academic Press

Key Words: composite particles; porous surface layer; sedimentation velocity; sedimentation potential; electrophoresis.

\section{INTRODUCTION}

The motions of charged colloidal particles in an electrolyte solution have received quite an amount of attention in the

${ }^{1}$ To whom correspondence should be addressed. past, due to their wide applications in the areas of chemical, biomedical, and environmental engineering and science. This problem is more complex than that of uncharged colloidal particles because the electric double layer surrounding each particle is distorted by the fluid flow around the particle. The deformation of the double layer resulting from the fluid motion is usually referred to as the relaxation effect and gives rise to an induced electric field. The sedimentation potential, which arises in a suspension of settling charged particles, was first reported by Dorn in 1878, and this phenomenon is often known by his name $(1,2)$. The sedimentation potential gradient (which is of the order $1-10 \mathrm{~V} / \mathrm{m}$ ) not only alters the velocity and pressure distributions in the fluid due to its action on the electrolyte ions but also retards the settling of the particles by an electrophoretic effect.

An important contribution to the sedimentation theory of a nonconducting spherical particle with arbitrary double layer thickness was made by Booth (1). He solved a set of electrokinetic differential equations using a perturbation method to obtain formulas for the sedimentation velocity and sedimentation potential expressed as power series in the zeta potential $(\zeta)$ of the particle up to $O\left(\zeta^{2}\right)$ and $O(\zeta)$, respectively. Using a unit cell model with the condition of zero net electric current, Levine et al. (3) derived analytical expressions of the sedimentation velocity and potential in a suspension of identical charged spheres with small surface potential as functions of the fractional volume concentration of the particles. In the limiting case of a single particle their result somewhat differs from that obtained by Booth, which is not subject to the constraint of zero net current. Numerical results relieving the restriction of low surface potential in Booth's analysis were reported by Stigter (4) using a modification of the theory of electrophoresis by Wiersema et al. (5). It was found that the Onsager reciprocal relation between the sedimentation potential and the electrophoretic mobility derived by de Groot et al. (6) is satisfied within good computational accuracy. Taking the double layer distortion from equilibrium as a small perturbation, Ohshima $e t$ al. (7) obtained general expressions and presented numerical results for the sedimentation velocity and potential of charged spheres over a broad range of zeta potential and double layer thickness. Other than cases of spherical parti- 
cles, the effect of the deformation of the ion cloud surrounding a charged circular cylinder on the sedimentation velocity of the particle has also been investigated semianalytically $(8,9)$.

The surface of a colloidal particle is generally not hard and smooth as assumed in many theoretical models. For instance, surface layers are purposely formed by adsorbing polymers to make the suspended particles stable against flocculation (10). Even the surfaces of model colloids such as silica and polystyrene latices are "hairy" with a gel-like polymeric layer extending from the bulk material inside the particle (11). In particular, the surface of a biological cell is not a hard smooth wall, but rather is a permeable rough surface with various appendages ranging from protein molecules on the order of nanometers to cilia on the order of microns (12). Such particles can be modeled as a composite particle having a central solid core and an outer porous shell (13). The creeping flow of an incompressible Newtonian fluid past a composite sphere was solved by Masliyah et al. (14) using the Brinkman equation for the flow field inside the fluid-permeable surface layer and the Stokes equations for the flow field external to the particle. An analytical formula for the drag force experienced by the particle was derived as a function of the radius of the solid core, the thickness of the porous shell, and the permeability of the shell. They also measured the settling velocities of a solid sphere with attached threads and found that theoretical predictions for the composite sphere are in excellent agreement with the experimental results. Employing the free-surface cell model for the creeping flow relative to an assemblage of identical composite spheres, Prasad et al. (15) later obtained an analytical solution for the dependence of the average drag force of this assemblage on the volume fraction of the particles.

Most of the past theoretical studies on the electrophoresis of a colloidal particle covered by a charged porous surface layer $(12,16-20)$ were performed with the assumption that the local radii of curvature of the particle are much larger than the thicknesses of the electric double layer and of the porous surface layer (i.e., the particle surface is planar and the applied electric field is parallel to it). Experimental results of the electrophoretic mobility of charged composite particles are also available for human erythrocytes (21), rat lymphocytes (22), and latex particles coated with poly $(\mathrm{N}$ isopropylacrylamide) hydrogel layers (23). Based on a formula derived from the theory of a planar particle surface, these experimental results could be used to calculate the fixed charge density and the hydrodynamic resistance parameter of the porous surface layer. Recently, a general expression for the electrophoretic mobility of a spherical composite particle was derived by Ohshima (24), neglecting the relaxation effect of the double layer. It has been found that the electrophoretic mobility of a charged composite sphere can be quite different from that of a "bare" rigid sphere. However, the effects of particle charges on the sedimentation velocity and potential of composite particles have not yet been theoretically investigated.

In this paper, the sedimentation in a dilute suspension of charged composite particles is studied. The densities of the fixed charges and the hydrodynamic frictional segments are assumed to be uniform throughout the porous surface layer of each particle, but no assumption is made as to the thicknesses of the double layer and of the surface layer relative to the dimension of the particle. In the next section, we present the fundamental electrokinetic equations and boundary conditions which govern the electrolyte ion distributions, the electrostatic potential profile, and the fluid flow field inside and outside the surface layer of a composite particle migrating in an unbounded solution. These basic equations are linearized assuming that the ion concentrations, the electric potential, and the fluid pressure have only a slight deviation from equilibrium due to the motion of the particle. In Section 3, the axisymmetric motion of a charged composite sphere in an unbounded solution of a symmetric electrolyte is considered. Using the Debye-Hückel approximation, we first get the solution of the equilibrium electric potential and ion concentration distributions. Then, the linearized electrokinetic equations are transformed into a set of differential equations by a perturbation method with the fixed charge densities of the porous surface layer and of the rigid core surface as the small perturbation parameters. The perturbed ion concentration, electric potential, fluid velocity, and pressure profiles are determined by solving this set of differential equations subject to the appropriate boundary conditions. A closed-form expression for the settling velocity of the charged composite sphere is obtained from a balance among its gravitational, electrostatic and hydrodynamic forces in Section 4. In Section 5, the average electric current density in a dilute suspension of identical charged composite spheres is derived, and an explicit formula for the sedimentation potential is resulted from letting the net current in the suspension be zero. The Onsager relation between sedimentation and electrophoresis is found to be satisfied for the composite spheres. Finally, analytical expressions in some limiting cases and typical numerical results of the sedimentation velocity and potential for charged composite spheres are presented in Section 6.

\section{BASIC ELECTROKINETIC EQUATIONS FOR THE MOTION OF A CHARGED COMPOSITE PARTICLE}

In this section we consider the relative motion of a charged composite particle of arbitrary shape in an unbounded liquid solution containing $M$ ionic species. The definition of a charged composite particle is a charged rigid particle core covered by a surface layer of charged porous substance or adsorbed polyelectrolytes in equilibrium with the surrounding electrolyte solution. The porous surface layer is treated as a solvent-permeable and ion-penetrable homogeneous shell in which fixed-charged groups are assumed to 
distribute at a uniform density. Conservation of all species, which do not react with one another, in the steady state requires that

$$
\nabla \cdot \mathbf{J}_{m}=0, \quad m=1,2, \ldots, M
$$

where $\mathbf{J}_{m}(\mathbf{x})$ is the number flux of species $m$ at position $\mathbf{x}$. If the solution is dilute, the flux can be given by

$$
\mathbf{J}_{m}=n_{m} \mathbf{u}-D_{m}\left(\nabla n_{m}+\frac{z_{m} e n_{m}}{k T} \nabla \psi\right),
$$

where $n_{m}(\mathbf{x})$ and $z_{m}$ are the concentration (number density) distribution and the valence of species $m$, respectively; $D_{m}$ is the diffusion coefficient of species $m$ which is assumed to be constant both inside and outside the surface layer; $\mathbf{u}(\mathbf{x})$ is the fluid velocity field relative to the particle; $\psi(\mathbf{x})$ is the electric potential distribution; $e$ is the elementary electric charge; $k$ is Boltzmann's constant; $T$ is the absolute temperature. The first term on the right-hand side of Eq. [2] represents the convection of the ionic species by the fluid and the second term denotes the diffusion and electrically induced migration of the species.

We assume that the Reynolds number of the fluid motion is very small, so the inertial effect on the fluid momentum balance can be neglected. The fluid flow is governed by a combination of the Stokes and Brinkman equations modified with the electrostatic effect,

$$
\begin{aligned}
\eta \nabla^{2} \mathbf{u}-\mathrm{h}(\mathbf{x}) f \mathbf{u} & =\nabla p-\rho \mathbf{g}+\rho_{e} \nabla \psi, \\
\nabla \cdot \mathbf{u} & =0,
\end{aligned}
$$

where $\eta$ and $\rho$ are the viscosity and density, respectively, of the fluid; $f$ is the friction coefficient inside the surface layer of the composite particle per unit volume of the fluid; $p(\mathbf{x})$ is the fluid pressure distribution, $\mathbf{g}$ is the acceleration of gravity, and $\rho_{e}(\mathbf{x})$ is the space charge density resulting from the mobile ionic species and can be written as

$$
\rho_{e}=\sum_{m=1}^{M} z_{m} e n_{m}
$$

In Eq. [3],$h(\mathbf{x})$ is a unit step function which equals unity if $\mathbf{x}$ is inside the surface layer and which equals zero if $\mathbf{x}$ is outside the composite particle; $\rho, \eta, f$, and $\mathbf{g}$ are assumed to be constant. Note that $f$ can be expressed as $6 \pi \eta a_{\mathrm{S}} N_{\mathrm{S}}$, where $N_{\mathrm{S}}$ and $a_{\mathrm{S}}$ are the number density and the Stokes radius, respectively, of the frictional segments in the surface layer.

The local electric potential $\psi$ and the charge density $\rho_{e}$ are related by Poisson's equation,

$$
\nabla^{2} \psi=-\frac{4 \pi}{\epsilon}\left[\rho_{e}+\mathrm{h}(\mathbf{x}) Q\right] .
$$

Here, $Q$ is the fixed charge density inside the surface layer; $\epsilon=4 \pi \epsilon_{0} \epsilon_{\mathrm{r}}$, where $\epsilon_{\mathrm{r}}$ is the relative permittivity of the electrolyte solution which is assumed to be the same inside and outside the surface layer and $\epsilon_{0}$ is the permittivity of a vacuum. Note that the space charge density in the surface layer is the sum of the densities of the mobile ions and the fixed charges.

The boundary conditions at the surface of the nonconducting rigid particle core are

$$
\begin{aligned}
\mathbf{u} & =\mathbf{0}, \\
\mathbf{n} \cdot \mathbf{J}_{m} & =0, \\
\mathbf{n} \cdot \nabla \psi & =-\frac{4 \pi}{\epsilon} \sigma,
\end{aligned}
$$

where $\mathbf{n}$ is the unit normal outward from the boundary surface, $\sigma$ is the surface charge density of the "bare" particle core. In Eq. [7a], we have assumed that the shear plane coincides with the surface of the rigid core. Equations [7b] and $[7 \mathrm{c}]$ state that no ions can penetrate into the rigid core, and the Gauss condition holds at the surface of the rigid core.

The conditions far from the particle are

$$
\begin{aligned}
\mathbf{u} & \rightarrow-\mathbf{U}, \\
n_{m} & \rightarrow n_{m}^{\infty}, \\
\psi & \rightarrow 0 .
\end{aligned}
$$

Here, $\mathbf{U}$ is the translational velocity of the particle, $n_{m}^{\infty}$ is the constant bulk concentration of type $m$ ions, and the electric potential in the bulk solution is set equal to zero. Equations [2], [3], and [8a] take a reference frame that the composite particle is at rest and the velocity of the fluid at infinity is the particle velocity in the opposite direction.

The boundary conditions at the surface of the composite particle (the boundary between the surface layer and the external solution, $S^{ \pm}$) are

$$
\begin{gathered}
\left.\mathbf{u}\right|_{\mathrm{s}^{+}}=\left.\mathbf{u}\right|_{\mathrm{s}^{-}}, \\
\left.\mathbf{n} \cdot \boldsymbol{\sigma}\right|_{\mathrm{s}^{+}}=\left.\mathbf{n} \cdot \boldsymbol{\sigma}\right|_{\mathrm{s}^{-}}, \\
\left.n_{m}\right|_{\mathrm{s}^{+}}=n_{m} \mid \mathrm{s}^{-}, \\
\left.\mathbf{J}_{m}\right|_{\mathrm{s}^{+}}=\left.\mathbf{J}_{m}\right|_{\mathrm{s}^{-}}, \\
\left.\psi\right|_{\mathrm{s}^{+}}=\left.\psi\right|_{\mathrm{s}^{-}}, \\
\left.\nabla \psi\right|_{\mathrm{s}^{+}}=\left.\nabla \psi\right|_{\mathrm{s}^{-}} .
\end{gathered}
$$


Here, $\boldsymbol{\sigma}(\mathbf{x})$ is the sum of the hydrodynamic stress $\boldsymbol{\sigma}^{\mathrm{H}}(\mathbf{x})$ and the Maxwell stress $\boldsymbol{\sigma}^{\mathrm{E}}(\mathbf{x})$ of the fluid given by

$$
\boldsymbol{\sigma}^{\mathrm{H}}=-p \mathbf{I}+\eta\left[\nabla \mathbf{u}+(\nabla \mathbf{u})^{\mathrm{T}}\right]
$$

and

$$
\boldsymbol{\sigma}^{\mathrm{E}}=\frac{\epsilon}{4 \pi}\left(\mathbf{E E}-\frac{1}{2} E^{2} \mathbf{I}\right)
$$

where $\mathbf{I}$ is the unit dyadic, $\mathbf{E}(\mathbf{x})=-\nabla \psi$ is the electric field, and $E=|\mathbf{E}|$. Equations [9a] and [9b] are the continuity requirement of the fluid velocity and stress tensor at the particle surface. Equations [9c] and [9d] state that the concentration and flux of species $m$ must be continuous. Equations [9e] and [9f] indicate that the potential and electric field are also continuous. The continuity of the electric field results from the assumption that the relative permittivity of the solution takes the same value both inside and outside the surface layer of the composite particle. From Eqs. [9b], [9f], and [10b], one obtains that both the Maxwell stress and the hydrodynamic stress must be continuous at the particle surface, viz., condition [9b] can be replaced by

$$
\left.\mathbf{n} \cdot \boldsymbol{\sigma}^{H}\right|_{\mathrm{s}^{+}}=\left.\mathbf{n} \cdot \boldsymbol{\sigma}^{H}\right|_{\mathrm{s}^{-}}
$$

Because the governing equations are coupled nonlinear partial differential equations, it is a formidable task to find a general solution of them. Therefore, we shall assume that the Peclet number is sufficiently small so that the system is only slightly distorted from the equilibrium state where the particle and fluid are at rest and replace these nonlinear equations by approximate linear equations. One can write

$$
\begin{gathered}
p=p^{(e q)}+\delta p, \\
n_{m}=n_{m}^{(e q)}+\delta n_{m}, \\
\psi=\psi^{(e q)}+\delta \psi,
\end{gathered}
$$

where $p^{(e q)}(\mathbf{x}), n_{m}^{(e q)}(\mathbf{x})$, and $\psi^{(e q)}(\mathbf{x})$ are the equilibrium distributions of pressure, concentration of species $m$, and electric potential, respectively, and $\delta p(\mathbf{x}), \delta n_{m}(\mathbf{x})$, and $\delta \psi(\mathbf{x})$ are the small perturbations to the equilibrium state. The quantities $p^{(e q)}, n_{m}^{(e q)}$, and $\psi^{(e q)}$ must also satisfy the above-mentioned governing equations and boundary conditions under the equilibrium state. Thus, the fundamental Eqs. [1], [3] , and [6] lead to the following equations at $\mathbf{u}=\mathbf{0}$ :

$$
\nabla \cdot\left(\nabla n_{m}^{(e q)}+\frac{z_{m} e n_{m}^{(e q)}}{k T} \nabla \psi^{(e q)}\right)=0, \quad m=1,2, \ldots, M
$$

$$
\mathbf{0}=\nabla p^{(e q)}-\rho \mathbf{g}-\frac{\epsilon}{4 \pi} \nabla^{2} \psi^{(e q)} \nabla \psi^{(e q)}-\mathrm{h}(\mathbf{x}) Q \nabla \psi^{(e q)},
$$

$$
\nabla^{2} \psi^{(e q)}=-\frac{4 \pi}{\epsilon}\left[\sum_{m=1}^{M} z_{m} e n_{m}^{(e q)}+\mathrm{h}(\mathbf{x}) Q\right]
$$

Integration of Eq. [13] shows that $n_{m}^{(e q)}$ is related to $\psi^{(e q)}$ by the Boltzmann distribution,

$$
n_{m}^{(e q)}=n_{m}^{\infty} \exp \left(-\frac{z_{m} e \psi^{(e q)}}{k T}\right) .
$$

A governing equation for $\psi^{(e q)}$ is obtained by the substitution of Eq. [16] into Eq. [15].

Substituting Eq. [12] into Eqs. [1], [3], and [6], using Eqs. [13]-[15], and neglecting the products of the small quantities $\mathbf{u}, \delta n_{m}$, and $\delta \psi$, one obtains

$$
\begin{aligned}
\mathbf{u} \cdot \nabla n_{m}^{(e q)} & -D_{m} \nabla \cdot\left[\nabla \delta n_{m}+\frac{z_{m} e}{k T}\left(n_{m}^{(e q)} \nabla \delta \psi\right.\right. \\
& \left.\left.+\delta n_{m} \nabla \psi^{(e q)}\right)\right]=0, \quad m=1,2, \ldots, M,
\end{aligned}
$$

$$
\begin{aligned}
& \nabla^{2} \mathbf{u}-\mathrm{h}(\mathbf{x}) \lambda^{2} \mathbf{u}= \frac{1}{\eta} \nabla \delta p-\frac{\epsilon}{4 \pi \eta}\left(\nabla^{2} \psi^{(e q)} \nabla \delta \psi\right. \\
&\left.+\nabla^{2} \delta \psi \nabla \psi^{(e q)}\right)-\frac{1}{\eta} \mathrm{h}(\mathbf{x}) Q \nabla \delta \psi \\
& \nabla^{2} \delta \psi=-\frac{4 \pi}{\epsilon} \sum_{m=1}^{M} z_{m} e \delta n_{m}
\end{aligned}
$$

where $\lambda=(f / \eta)^{1 / 2}$. Note that the reciprocal of the parameter $\lambda$ is the shielding length characterizing the extent of flow penetration inside the surface layer of the composite particle.

The conditions for $\delta n_{m}$ and $\delta \psi$ that resulted from Eqs. [7] and $[8]$ are

$\mathbf{n} \cdot \nabla \delta n_{m}$ and $\mathbf{n} \cdot \nabla \delta \psi$ at the rigid core surface

of the composite particle are zero,

$$
\delta n_{m} \text { and } \delta \psi \text { at infinity must be zero. }
$$

Equations [9c]-[9f] yield the following boundary conditions at the surface of the composite particle:

$$
\begin{aligned}
\left.\delta n_{m}\right|_{\mathrm{s}^{+}} & =\left.\delta n_{m}\right|_{\mathrm{s}^{-}}, \\
\left.\nabla \delta n_{m}\right|_{\mathrm{s}^{+}} & =\left.\nabla \delta n_{m}\right|_{\mathrm{s}^{-}}, \\
\left.\delta \psi\right|_{\mathrm{s}^{+}} & =\left.\delta \psi\right|_{\mathrm{s}^{-}}, \\
\left.\nabla \delta \psi\right|_{\mathrm{s}^{+}} & =\left.\nabla \delta \psi\right|_{\mathrm{s}^{-}} .
\end{aligned}
$$




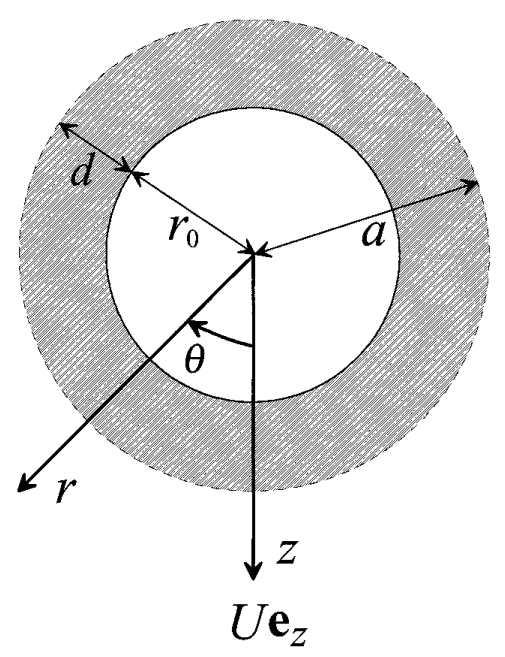

FIG. 1. Geometrical sketch for the sedimentation of a composite sphere.

The fluid velocity $\mathbf{u}$ is a small perturbed quantity, and the boundary conditions for $\mathbf{u}$ have been given by Eqs. [7a], [8a], [9a], and [11].

\section{SOLUTION FOR THE MOTION OF A CHARGED COMPOSITE SPHERE IN SYMMETRIC ELECTROLYTES}

We now consider the axisymmetric motion of a charged composite sphere of radius $a$ immersed into an unbounded solution of a symmetrically charged, binary electrolyte with a constant bulk concentration $n^{\infty}\left(M=2, z_{+}=-z_{-}=z\right.$, $n_{+}^{\infty}=n_{-}^{\infty}=n^{\infty}$, where subscripts + and - refer to the cation and anion, respectively). As illustrated in Fig. 1, the composite particle has a porous surface layer of constant thickness $d$ so that the radius of the rigid core is $r_{0}=a-d$. The translational velocity of the particle $\mathbf{U}=U \mathbf{e}_{z}$, where $\mathbf{e}_{z}$ is the unit vector in the axial direction. The origin of the spherical coordinate system $(r, \theta, \phi)$ is taken at the center of the particle.

We first seek the solution of $\psi^{(e q)}$ for a charged composite sphere. Substituting the Boltzmann distribution, Eq. [16], into Poisson's equation [15] for a symmetric electrolyte, one obtains the equilibrium Poisson-Boltzmann equation,

$$
\nabla^{2} \psi^{(e q)}=\frac{k T}{z e} \kappa^{2} \sinh \left(\frac{z e \psi^{(e q)}}{k T}\right)-\mathrm{h}(r) \frac{4 \pi Q}{\epsilon} .
$$

Here, $\mathrm{h}(r)$ equals unity if $r_{0}<r<a$ and is zero if $r>a$; $\kappa$ is the Debye-Hückel parameter defined by

$$
\kappa=\left(\frac{8 \pi z^{2} e^{2} n^{\infty}}{\epsilon k T}\right)^{1 / 2}
$$

The boundary conditions for $\psi^{(e q)}$ resulted from Eqs. [7c], $[8 \mathrm{c}],[9 \mathrm{e}]$, and [9f] are

$$
\begin{aligned}
\left.\frac{d \psi^{(e q)}}{d r}\right|_{r=r_{0}} & =-\frac{4 \pi \sigma}{\epsilon}, \\
\left.\psi^{(e q)}\right|_{r=a^{+}} & =\left.\psi^{(e q)}\right|_{r=a^{-}}, \\
\left.\frac{d \psi^{(e q)}}{d r}\right|_{r=a^{+}} & =\left.\frac{d \psi^{(e q)}}{d r}\right|_{r=a^{-}}, \\
\left.\psi^{(e q)}\right|_{r \rightarrow \infty} \rightarrow 0 . &
\end{aligned}
$$

The solution to Eq. [23] satisfying Eq. [25] is

$$
\psi^{(e q)}=\psi_{e q 01}(r) \bar{\sigma}+\psi_{e q 10}(r) \bar{Q}+O\left(\bar{\sigma}^{3}, \bar{\sigma}^{2} \bar{Q}, \bar{\sigma} \bar{Q}^{2}, \bar{Q}^{3}\right)
$$

where

$$
\bar{\sigma}=\frac{4 \pi z e \sigma}{\epsilon \kappa k T}
$$

and

$$
\bar{Q}=\frac{4 \pi z e Q}{\epsilon \kappa^{2} k T},
$$

which are the nondimensional charge densities of the rigid core surface and of the surface layer, respectively, of the composite particle, and

$$
\begin{gathered}
\left.\psi_{e q 01}(r)=\frac{k T}{z e}\left(\frac{\kappa r_{0}}{1+\kappa r_{0}}\right) \frac{r_{0}}{r} e^{-\kappa\left(r-r_{0}\right)} ; \quad \text { [28a }\right] \\
\psi_{e q 0}(r)=\frac{k T}{z e}\left\{1-\left(1+\frac{1}{\kappa a}\right) \frac{e^{-\kappa d}}{1+\kappa r_{0}}\right. \\
\left.\times\left[\kappa r_{0} \cosh \left(\kappa r-\kappa r_{0}\right)+\sinh \left(\kappa r-\kappa r_{0}\right)\right] \frac{a}{r}\right\}, \\
\psi_{e q 10}(r)=\frac{k T}{z e}\left\{1-\left(1+\frac{1}{\kappa a}\right) \frac{e^{-\kappa d}}{1+\kappa r_{0}}\left[\kappa r_{0} \cosh (\kappa d)\right.\right. \\
+\sinh (\kappa d)]\} \frac{a}{r} e^{-\kappa(r-a)}, \quad \text { if } r>a . \quad[28 \mathrm{c}]
\end{gathered}
$$

Note that $\psi^{(e q)}$ and $n_{ \pm}^{(e q)}$ depend on $r$ only due to spherical symmetry and that the function $\psi_{e q 01}(r)$ takes the same form both in the regions $r_{0}<r<a$ and $r>a$. Expression [26] for $\psi^{(e q)}$ as a power series in the fixed charge densities of the composite sphere up to $O(\bar{\sigma}, \bar{Q})$ is the equilibrium solution for the linearized Poisson-Boltzmann equation that is valid for small values of the electric potential (the DebyeHückel approximation). That is, the charge densities $\sigma$ and 
$Q$ of the composite particle must be small enough for the potential to remain small. Note that, the contribution from the effects of $O\left(\bar{\sigma}^{2}, \bar{\sigma} \bar{Q}, \bar{Q}^{2}\right)$ to $\psi^{(e q)}$ in Eq. [26] disappears only for the case of symmetric electrolytes.

To solve the small quantities $\delta n_{ \pm}, \mathbf{u}, \delta p$, and $\delta \psi$ in terms of the particle velocity $U$ when the parameters $\bar{\sigma}$ and $\bar{Q}$ are small, these variables can be written as perturbation expansions in powers of $\bar{\sigma}$ and $\bar{Q}$,

$$
\begin{aligned}
\delta n_{ \pm}= & n_{00 \pm}+n_{01 \pm} \bar{\sigma}+n_{10 \pm} \bar{Q}+n_{02 \pm} \bar{\sigma}^{2} \\
& +n_{11 \pm} \bar{\sigma} \bar{Q}+n_{20 \pm} \bar{Q}^{2}+\cdots, \\
\mathbf{u}= & \mathbf{u}_{00}+\mathbf{u}_{01} \bar{\sigma}+\mathbf{u}_{10} \bar{Q}+\mathbf{u}_{02} \bar{\sigma}^{2} \\
& +\mathbf{u}_{11} \bar{\sigma} \bar{Q}+\mathbf{u}_{20} \bar{Q}^{2}+\cdots, \\
\delta p= & p_{00}+p_{01} \bar{\sigma}+p_{10} \bar{Q}+p_{02} \bar{\sigma}^{2} \\
& +p_{11} \bar{\sigma} \bar{Q}+p_{20} \bar{Q}^{2}+\cdots, \\
\delta \psi= & \psi_{00}+\psi_{01} \bar{\sigma}+\psi_{10} \bar{Q}+\psi_{02} \bar{\sigma}^{2} \\
& +\psi_{11} \bar{\sigma} \bar{Q}+\psi_{20} \bar{Q}^{2}+\cdots, \\
U= & U_{00}+U_{01} \bar{\sigma}+U_{10} \bar{Q}+U_{02} \bar{\sigma}^{2} \\
& +U_{11} \bar{\sigma} \bar{Q}+U_{20} \bar{Q}^{2}+\cdots,
\end{aligned}
$$

where the functions $n_{i j \pm}, \mathbf{u}_{i j}, p_{i j}, \psi_{i j}$, and $U_{i j}$ are independent of $\bar{\sigma}$ and $\bar{Q}$. Both $n_{00 \pm}$ and $\psi_{00}$ must equal zero due to not imposing the concentration gradient and electric field.

Substituting the expansions given by Eq. [29] and $\psi^{(e q)}$ and $n_{ \pm}^{(e q)}$ given by Eqs. [26] and [16] into the governing equations [17]-[19] and boundary conditions [7a], [8a], [9a], [11], [20], [21], and [22], and equating like powers of $\bar{\sigma}$ and $\bar{Q}$ on both sides of the respective equations, we obtain a set of linear differential equations and boundary conditions for each set of the functions $n_{i j \pm}, \mathbf{u}_{i j}, p_{i j}$, and $\psi_{i j}$ with $i$ and $j$ equal to $0,1,2, \ldots$ These perturbation equations and their solutions are presented in the Appendix, and the results for the $r$ and $\theta$ components of $\mathbf{u}, \delta p$ (to the orders of $\bar{\sigma}^{2}, \bar{\sigma} \bar{Q}$, and $\bar{Q}^{2}$ ), $\delta \psi$, and $\delta \mu_{ \pm}$(to the orders of $\bar{\sigma}$ and $\bar{Q})$ can be written as

$$
\begin{aligned}
u_{r}= & \left\{U_{00} F_{00 r}(r)+U_{01} F_{00 r}(r) \bar{\sigma}+U_{10} F_{00 r}(r) \bar{Q}\right. \\
& +\left[U_{02} F_{00 r}(r)+U_{00} F_{02 r}(r)\right] \bar{\sigma}^{2}+\left[U_{11} F_{00 r}(r)\right. \\
& \left.+U_{00} F_{11 r}(r)\right] \bar{\sigma} \bar{Q}+\left[U_{20} F_{00 r}(r)\right. \\
& \left.\left.+U_{00} F_{20 r}(r)\right] \bar{Q}^{2}\right\} \cos \theta, \\
u_{\theta}= & \left\{U_{00} F_{00 \theta}(r)+U_{01} F_{00 \theta}(r) \bar{\sigma}+U_{10} F_{00 \theta}(r) \bar{Q}\right. \\
& +\left[U_{02} F_{00 \theta}(r)+U_{00} F_{02 \theta}(r)\right] \bar{\sigma}^{2}+\left[U_{11} F_{00 \theta}(r)\right. \\
& \left.+U_{00} F_{11 \theta}(r)\right] \bar{\sigma} \bar{Q}+\left[U_{20} F_{00 \theta}(r)\right. \\
& \left.\left.+U_{00} F_{20 \theta}(r)\right] \bar{Q}^{2}\right\} \sin \theta,
\end{aligned}
$$

$$
\begin{aligned}
\delta p= & \frac{\eta}{a}\left\{U_{00} F_{P 00}(r)+U_{01} F_{P 00}(r) \bar{\sigma}+U_{10} F_{P 00}(r) \bar{Q}\right. \\
& +\left[U_{02} F_{P 00}(r)+U_{00} F_{P 02}(r)\right. \\
& \left.+\frac{a \epsilon \kappa^{2}}{4 \pi \eta} U_{00} \psi_{e q 01}(r) F_{\psi 01}(r)\right] \bar{\sigma}^{2} \\
& +\left[U_{11} F_{P 00}(r)+U_{00} F_{P 11}(r)+\frac{a \epsilon \kappa^{2}}{4 \pi \eta} U_{00}\right. \\
& \left.\times\left(\psi_{e q 01}(r) F_{\psi 10}(r)+\psi_{e q 10}(r) F_{\psi 01}(r)\right)\right] \bar{\sigma} \bar{Q} \\
& +\left[U_{20} F_{P 00}(r)+U_{00} F_{P 20}(r)\right. \\
& \left.\left.+\frac{a \epsilon \kappa^{2}}{4 \pi \eta} U_{00} \psi_{e q 10}(r) F_{\psi 10}(r)\right] \bar{Q}^{2}\right\} \cos \theta, \\
\delta \psi= & U_{00}\left[F_{\psi 01}(r) \bar{\sigma}+F_{\psi 10}(r) \bar{Q}\right] \cos \theta, \\
\delta \mu_{ \pm}= & U_{00}\left[F_{01 \pm}(r) \bar{\sigma}+F_{10 \pm}(r) \bar{Q}\right] \cos \theta .
\end{aligned}
$$

Here, $\delta \mu_{ \pm}$is defined as a linear combination of $\delta n_{ \pm}$and $\delta \psi$ based on the concept of the electrochemical potential (7),

$$
\delta \mu_{ \pm}=\frac{k T}{z e n^{\infty}} \delta n_{ \pm} \pm \delta \psi
$$

and $F_{i j r}(r), F_{i j \theta}(r), F_{P i j}(r), F_{\psi i j}(r)$, and $F_{i j \pm}(r)$ with $i$ and $j$ equal to 0,1 , and 2 are functions of $r$ given by Eqs. [A4], [A19], [A20], and [A26] in the Appendix.

\section{SEDIMENTATION VELOCITY OF A CHARGED COMPOSITE SPHERE}

The total force exerted on a charged composite sphere of radius $a$ settling in an electrolyte solution can be expressed as

$$
\mathbf{F}_{\text {total }}=\mathbf{F}_{\mathrm{g}}+\mathbf{F}_{\mathrm{e}}+\mathbf{F}_{\mathrm{h}}
$$

Here, $\mathbf{F}_{\mathrm{g}}$ is the gravitational force (and buoyant force), $\mathbf{F}_{\mathrm{e}}$ is the electric force, and $\mathbf{F}_{\mathrm{h}}$ is the hydrodynamic drag force acting on the particle.

The gravitational force is given by

$$
\mathbf{F}_{\mathrm{g}}=V_{\mathrm{t}}\left[\gamma_{\mathrm{p}}\left(\rho_{\mathrm{p}}-\rho\right)+\gamma_{\mathrm{c}}\left(\rho_{\mathrm{c}}-\rho\right)\right] g \mathbf{e}_{z},
$$

where $V_{\mathrm{t}}$ is the dry volume of the composite particle, $\rho_{\mathrm{p}}$ and $\rho_{\mathrm{c}}$ are, respectively, the true densities of the surface layer and the rigid core of the composite particle, $\gamma_{\mathrm{p}}$ and $\gamma_{\mathrm{c}}$ are the dry volume fractions of the surface layer and the rigid core, respectively, and $g \mathbf{e}_{z}=\mathbf{g}$ is the gravitational acceleration. 
The electric force acting on the charged composite sphere where is given by

$$
\mathbf{F}_{\mathrm{e}}=\int_{r=a} \boldsymbol{\sigma}^{\mathrm{E}} \cdot \mathbf{n} d S .
$$

Using the divergence theorem, the above equation can be written as

$$
\mathbf{F}_{\mathrm{e}}=-\int_{V_{\mathrm{A}}} \nabla \cdot \boldsymbol{\sigma}^{\mathrm{E}} d V+\int_{S_{\mathrm{A}}} \boldsymbol{\sigma}^{\mathrm{E}} \cdot \mathbf{n} d S,
$$

where $S_{\mathrm{A}}$ is an arbitrary close surface surrounding the composite particle and $V_{\mathrm{A}}$ is the volume between the particle surface and $S_{\mathrm{A}}$. Since the electric potential is screened by the ions around the particle and decays exponentially, one can choose $S_{\mathrm{A}}$ to be a spherical surface with sufficiently large radius so that the second term on the right-hand side of Eq. [37] is negligibly small. Thus, the electric force can be represented by the integral over the fluid volume outside the composite sphere. Substitution of the Maxwell stress given by Eq. [10b] into Eq. [37] with this arrangement produces

$$
\mathbf{F}_{\mathrm{e}}=-\frac{\epsilon}{4 \pi} \int_{r>a} \nabla \psi \nabla^{2} \psi d V
$$

Due to the net electric force acting on the particle at the equilibrium state is zero, the leading order of the electric force is given by

$$
\mathbf{F}_{\mathrm{e}}=-\frac{\epsilon}{4 \pi} \int_{r>a}\left(\nabla \delta \psi \nabla^{2} \psi^{(e q)}+\nabla \psi^{(e q)} \nabla^{2} \delta \psi\right) d V
$$

Substituting Eqs. [26] and [31] into Eq. [39], one has

$$
\begin{aligned}
\mathbf{F}_{\mathrm{e}}= & \left\{\left[\frac{\epsilon(\kappa a)^{2}}{3} \psi_{e q 01}(a) F_{\psi 01}(a)\right.\right. \\
& \left.+4 \pi \eta a \int_{a}^{\infty}\left(\frac{r}{a}\right)^{3} G_{02}(r) d r\right] U_{00} \bar{\sigma}^{2} \\
& +\left[\frac{\epsilon(\kappa a)^{2}}{3}\left(\psi_{e q 01}(a) F_{\psi 01}(a)+\psi_{e q 10}(a) F_{\psi 01}(a)\right)\right. \\
& \left.+4 \pi \eta a \int_{a}^{\infty}\left(\frac{r}{a}\right)^{3} G_{11}(r) d r\right] U_{00} \bar{\sigma} \bar{Q} \\
& +\left[\frac{\epsilon(\kappa a)^{2}}{3} \psi_{e q 10}(a) F_{\psi 10}(a)\right. \\
& \left.+4 \pi \eta a \int_{a}^{\infty}\left(\frac{r}{a}\right)^{3} G_{20}(r) d r\right] U_{00} \bar{Q}^{2} \\
& \left.+O\left(\bar{\sigma}^{3}, \bar{\sigma}^{2} \bar{Q}, \bar{\sigma} \bar{Q}^{2}, \bar{Q}^{3}\right)\right\} \mathbf{e}_{z},
\end{aligned}
$$

In Eq. [41], functions $\psi_{e q 01}(r)$ and $\psi_{e q 10}(r)$ have been given by Eq. [28], and $F_{01 \pm}(r)$ and $F_{10 \pm}(r)$ are defined by Eq. [A19] in the Appendix.

The hydrodynamic drag force acting on the composite sphere is given by

$$
\mathbf{F}_{\mathrm{h}}=\int_{r=a}\left\{-\delta p \mathbf{I}+\eta\left[\nabla \mathbf{u}+(\nabla \mathbf{u})^{T}\right]\right\} \cdot \mathbf{n} d S .
$$

Substitution of Eq. [30] into the above equation results in

$$
\begin{aligned}
\mathbf{F}_{\mathrm{h}}= & -\left\{4 \pi \eta a C_{006}\left(U_{00}+U_{01} \bar{\sigma}+U_{10} \bar{Q}\right)\right. \\
& +\left[4 \pi \eta a\left(C_{006} U_{02}+C_{026} U_{00}\right)\right. \\
& \left.+\frac{\epsilon(\kappa a)^{2}}{3} U_{00} \psi_{e q 01}(a) F_{\psi 01}(a)\right] \bar{\sigma}^{2} \\
& +\left[4 \pi \eta a\left(C_{006} U_{11}+C_{116} U_{00}\right)+\frac{\epsilon(\kappa a)^{2}}{3}\right. \\
& \left.\times U_{00}\left(\psi_{e q 01}(a) F_{\psi 10}(a)+\psi_{e q 10}(a) F_{\psi 01}(a)\right)\right] \bar{\sigma} \bar{Q} \\
& +\left[4 \pi \eta a\left(C_{006} U_{20}+C_{206} U_{00}\right)\right. \\
& \left.+\frac{\epsilon(\kappa a)^{2}}{3} U_{00} \psi_{e q 10}(a) F_{\psi 10}(a)\right] \bar{Q}^{2} \\
& \left.+O\left(\bar{\sigma}^{3}, \bar{\sigma}^{2} \bar{Q}, \bar{\sigma} \bar{Q}^{2}, \bar{Q}^{3}\right)\right\} \mathbf{e}_{z},
\end{aligned}
$$

where coefficients $C_{006}, C_{026}, C_{116}$, and $C_{206}$ are given by Eqs. [A6f] and [A27f].

At the steady state, the total force acting on the settling particle is zero. Using this constraint after the substitution of Eqs. [35], [40], and [43] for a symmetric electrolyte into Eq. [34], one obtains

$$
U_{00}=\frac{V_{\mathrm{t}}\left[\gamma_{\mathrm{p}}\left(\rho_{\mathrm{p}}-\rho\right)+\gamma_{\mathrm{c}}\left(\rho_{\mathrm{c}}-\rho\right)\right] g}{4 \pi \eta a C_{006}},
$$




$$
\begin{aligned}
& U_{01}=0, \\
& U_{10}=0, \\
& U_{02}=\frac{U_{00}}{C_{006}}\left[-C_{026}+\int_{a}^{\infty}\left(\frac{r}{a}\right)^{3} G_{02}(r) d r\right], \\
& U_{11}=\frac{U_{00}}{C_{006}}\left[-C_{116}+\int_{a}^{\infty}\left(\frac{r}{a}\right)^{3} G_{11}(r) d r\right], \\
& U_{20}=\frac{U_{00}}{C_{006}}\left[-C_{206}+\int_{a}^{\infty}\left(\frac{r}{a}\right)^{3} G_{20}(r) d r\right] .
\end{aligned}
$$

Here, $U_{00}$ is the sedimentation velocity of an uncharged composite sphere, which was obtained by Masliyah et al. (14), and $C_{006}$ is a function of parameters $\lambda a$ and $r_{0} / a$ only. The definite integrals in the closed-form Eqs. [44d], [44e], and [44f] as well as in coefficients $C_{026}, C_{116}$, and $C_{206}$ can be performed numerically using Eqs. [A27f] and [41].

From Eqs. [29e] and [44], the settling velocity of the composite sphere can be expressed as

$$
\begin{aligned}
U= & U_{00}\left[1-(\kappa a)^{2} H_{1} \bar{\sigma}^{2}-(\kappa a)^{3} H_{2} \bar{\sigma} \bar{Q}\right. \\
& \left.-(\kappa a)^{4} H_{3} \bar{Q}^{2}+O\left(\bar{\sigma}^{3}, \bar{\sigma}^{2} \bar{Q}, \bar{\sigma} \bar{Q}^{2}, \bar{Q}^{3}\right)\right],
\end{aligned}
$$

where the dimensionless coefficients

$$
\begin{aligned}
& H_{1}=-\frac{U_{02}}{(\kappa a)^{2} U_{00}}, \\
& H_{2}=-\frac{U_{11}}{(\kappa a)^{3} U_{00}}, \\
& H_{3}=-\frac{U_{20}}{(\kappa a)^{4} U_{00}},
\end{aligned}
$$

and their numerical results calculated by using Eqs. [44a], [44d], [44e], and [44f] will be presented in Section 6. Note that $(\kappa a)^{2} \bar{\sigma}^{2},(\kappa a)^{3} \bar{\sigma} \bar{Q}$, and $(\kappa a)^{4} \bar{Q}^{2}$ are independent of $\kappa$ or $n^{\infty}$. For a given electrolyte solution, coefficients $H_{1}, H_{2}$, and $H_{3}$ are functions of parameters $\kappa a, \lambda a$, and $r_{0} / a$ only.

\section{SEDIMENTATION POTENTIAL IN A SUSPENSION OF CHARGED COMPOSITE SPHERES}

We now consider the steady-state sedimentation of a dilute suspension of identical charged composite spheres of radius $a$ in a solution containing $M$ ionic species. The electric fields around the individual particles collectively give a sedimentation field $\mathbf{E}_{\mathrm{SED}}$ in the suspension. It is assumed that the suspension is statistically homogeneous, and all effects of its boundaries are ignored. $\mathbf{E}_{\mathrm{SED}}$ is then uniform in the suspension and can be regarded as the average of the gradient of electric potential over a sufficiently large volume $V$ of the suspension to contain many particles. Namely,

$$
\mathbf{E}_{\mathrm{SED}}=-\frac{1}{V} \int_{V} \nabla \psi d \mathbf{x}=-\frac{1}{V} \int_{V} \nabla \delta \psi d \mathbf{x},
$$

where we have used Eq. [12c] and the fact that the volume average of the gradient of the equilibrium electric potential is zero. In order to calculate $\mathbf{E}_{\mathrm{SED}}$, we use the requirement that there exists no net current in the suspension. That is, the volume-average current density,

$$
\langle\mathbf{i}\rangle=\frac{1}{V} \int_{V} \mathbf{i} d \mathbf{x}
$$

must be zero. In Eq. [48], $\mathbf{i}(\mathbf{x})$ is the current density distribution. The mathematical analysis given below is similar to that developed by O'Brien (25) and Ohshima et al. (7).

The current density $\mathbf{i}$ can be written as

$$
\mathbf{i}=\sum_{m=1}^{M} z_{m} e \mathbf{J}_{m} .
$$

Substituting Eqs. [2], [12], and [16] into the above equation and neglecting products of the small perturbation quantities, one has

$$
\begin{aligned}
\mathbf{i}=\mathbf{u} & \sum_{m=1}^{M} z_{m} e n_{m}^{(e q)}-\sum_{m=1}^{M} z_{m} e D_{m} \\
& \times\left[\nabla \delta n_{m}+\frac{z_{m} e}{k T}\left(n_{m}^{(e q)} \nabla \delta \psi+\delta n_{m} \nabla \psi^{(e q)}\right] .\right.
\end{aligned}
$$

Far from any particle (beyond the double layer), $n_{m}^{(e q)} \rightarrow$ $n_{m}^{\infty}, \nabla \psi^{(e q)} \rightarrow \mathbf{0}$, and Eq. [50] becomes

$$
\mathbf{i} \rightarrow-\sum_{m=1}^{M} z_{m} e D_{m}\left(\nabla \delta n_{m}+\frac{z_{m} e n_{m}^{\infty}}{k T} \nabla \delta \psi\right) .
$$

By adding and subtracting the current density given by the above equation in the integrand of Eq. [48], one obtains

$$
\begin{aligned}
\langle\mathbf{i}\rangle= & -\sum_{m=1}^{M} \frac{z_{m} e D_{m}}{V} \int_{V}\left(\nabla \delta n_{m}+\frac{z_{m} e n_{m}^{\infty}}{k T} \nabla \delta \psi\right) d \mathbf{x} \\
& +\frac{1}{V} \int_{V}\left[\mathbf{i}+\sum_{m=1}^{M} z_{m} e D_{m}\left(\nabla \delta n_{m}+\frac{z_{m} e n_{m}^{\infty}}{k T} \nabla \delta \psi\right)\right] d \mathbf{x} .
\end{aligned}
$$

Note that the magnitude of $\mathbf{i}$ and $D_{m}$ can be taken as zero inside the dielectric rigid core of each composite sphere.

In a statistically homogenous suspension with constant bulk ionic concentrations, the volume average of $\nabla \delta n_{m}$ is zero. According to the definition of Eq. [47] the first term on the right-hand side of Eq. [52] can be written as 
$-\sum_{m=1}^{M} \frac{z_{m} e D_{m}}{V} \int_{V}\left(\nabla \delta n_{m}+\frac{z_{m} e n_{m}^{\infty}}{k T} \nabla \delta \psi\right) d \mathbf{x}=\Lambda^{\infty} \mathbf{E}_{\mathrm{SED}}$.

Here,

$$
\Lambda^{\infty}=\sum_{m=1}^{M} \frac{z_{m}^{2} e^{2} n_{m}^{\infty} D_{m}}{k T},
$$

which is the electric conductivity of the electrolyte solution in the absence of the particles. The integral in the second term on the right-hand side of Eq. [52] can be calculated by first considering for a single particle as if the others were absent and then multiplying the result by the particle number $N$ in $V$, since the integrand vanishes beyond the double layers surrounding the particles and the suspension is assumed to be dilute. Also, the volume integral can be transformed into a surface integral over a spherical boundary of infinite radius containing the single particle at its center. Thus, the second term becomes

$$
\begin{aligned}
& \frac{N}{V} \int_{r \rightarrow \infty} {\left[\mathbf{n} \cdot \mathbf{i r}+\sum_{m=1}^{M} z_{m} e D_{m}\left(\delta n_{m}+\frac{z_{m} e n_{m}^{\infty}}{k T} \delta \psi\right) \mathbf{n}\right] d S } \\
&=-\frac{\mathrm{N}}{\mathrm{V}} \sum_{m=1}^{M} z_{m} e D_{m} \int_{r \rightarrow \infty}\left[\mathbf{r}\left(\nabla \delta n_{m}+\frac{z_{m} e n_{m}^{\infty}}{k T} \nabla \delta \psi\right)\right. \\
&\left.\times \mathbf{n}-\left(\delta n_{m}+\frac{z_{m} e n_{m}^{\infty}}{k T} \delta \psi\right) \mathbf{n}\right] d S
\end{aligned}
$$

where $\mathbf{r}$ is the position vector relative to the particle center. To obtain Eq. [55], the requirement of the conservation of electric charge $(\nabla \cdot \mathbf{i}=0)$ and Eq. [51] have been used.

For a symmetric electrolyte with the absolute value of valence $z$, the average current density is obtained by the substitution of Eqs. [33], [53], and [55] into Eq. [52],

$$
\begin{array}{r}
\langle\mathbf{i}\rangle=\Lambda^{\infty} \mathbf{E}_{\mathrm{SED}}-\frac{z^{2} e^{2} n^{\infty} N}{k T V} \int_{r \rightarrow \infty}\left[D_{+}\left(\mathbf{r} \nabla \delta \mu_{+} \cdot \mathbf{n}-\delta \mu_{+} \mathbf{n}\right)\right. \\
\left.-D_{-}\left(\mathbf{r} \nabla \delta \mu_{-} \cdot \mathbf{n}-\delta \mu_{-} \mathbf{n}\right)\right] d S .
\end{array}
$$

Substituting Eq. [32] into Eq. [56] and using the requirement that $\langle\mathbf{i}\rangle=\mathbf{0}$, one has

$$
\begin{aligned}
\mathbf{E}_{\mathrm{SED}}= & \lim _{r \rightarrow \infty} \frac{4 \pi r^{2} z^{2} e^{2} n^{\infty} N U_{00}}{3 k T V \Lambda^{\infty}}\left\{\left[D_{+}\left(r \frac{d F_{01+}}{d r}-F_{01+}\right)\right.\right. \\
& \left.-D_{-}\left(r \frac{d F_{01-}}{d r}-F_{01-}\right)\right] \bar{\sigma}+\left[D _ { + } \left(r \frac{d F_{10+}}{d r}\right.\right. \\
& \left.\left.-F_{10+}\right)-D_{-}\left(r \frac{d F_{10-}}{d r}-F_{10-}\right)\right] \bar{Q} \\
& \left.+O\left(\bar{\sigma}^{3}, \bar{\sigma}^{2} \bar{Q}, \bar{\sigma} \bar{Q}^{2}, \bar{Q}^{3}\right)\right\} \mathbf{e}_{z} .
\end{aligned}
$$

Substituting Eqs. [A19] and [44a] into the above equation and making relevant calculations, we obtain the sedimentation potential field in a dilute suspension of identical charged composite spheres,

$$
\mathbf{E}_{\mathrm{SED}}=-\varphi\left[\gamma_{\mathrm{p}}\left(\rho_{\mathrm{p}}-\rho\right)+\gamma_{c}\left(\rho_{c}-\rho\right)\right] \frac{\mu_{\mathrm{E}}}{\Lambda^{\infty}} \mathbf{g}
$$

Here, $\varphi=N V_{t} / V$ is the true volume fraction of the composite particles and $\mu_{\mathrm{E}}$ is the electrophoretic mobility of a charged composite sphere of radius $a$ at low electric potential,

$$
\mu_{\mathrm{E}}=H_{\sigma} \frac{a \sigma}{\eta}+H_{Q} \frac{a^{2} Q}{\eta}+O\left(\bar{\sigma}^{3}, \bar{\sigma}^{2} \bar{Q}, \bar{\sigma} \bar{Q}^{2}, \bar{Q}^{3}\right),
$$

where the dimensionless coefficients $H_{\sigma}$ and $H_{Q}$ have the following closed forms:

$$
\begin{aligned}
& H_{\sigma}=\frac{\kappa z e}{6 a^{2} C_{006} k T} \int_{r_{0}}^{\infty}\left(2 r^{3}+r_{0}^{3}\right) F_{00 r}(r) \frac{d \psi_{e q 01}}{d r} d r, \\
& H_{Q}=\frac{z e}{6 a^{3} C_{006} k T} \int_{r_{0}}^{\infty}\left(2 r^{3}+r_{0}^{3}\right) F_{00 r}(r) \frac{d \psi_{e q 10}}{d r} d r .
\end{aligned}
$$

In Eq. [60], functions $\psi_{e q 01}(r)$ and $\psi_{e q 10}(r)$ are given by Eq. [28] and $F_{00 r}(r)$ is defined by Eqs. [A4a] and [A4d]. Again, the contribution from the second-order effects of the fixed charge densities of the composite spheres to $\mathbf{E}_{\mathrm{SED}}$ and $\mu_{\mathrm{E}}$ in Eqs. [57] and [59] vanishes only for symmetric electrolytes. The numerical results of coefficients $H_{\sigma}$ and $H_{Q}$ calculated from Eq. [60] will be presented in Section 6. For a specific electrolyte solution, these coefficients are functions of parameters $\kappa a, \lambda a$, and $r_{0} / a$ only. It is understood that the result given by Eqs. [58]-[60] is only valid with the requirements that $\varphi \ll 1$ and $\kappa a \varphi^{-1 / 3} \gg 1$.

Equation [58] is an Onsager reciprocal relation connecting the sedimentation potential with the electrophoretic mobility (correct to order $\varphi$ ) derived by de Groot et al. (6) on the basis of irreversible thermodynamics. For a dilute suspension of impermeable rigid spheres, this relation has also been demonstrated by Ohshima et al. (7). For the situation of combined sedimentation and electrophoresis in a dilute suspension of colloidal particles, the average electric current density $\langle\mathbf{i}\rangle$ and mass flux $\langle\mathbf{j}\rangle$ can be expressed in terms of the Onsager transport coefficients $b_{11}, b_{12}, b_{21}$, and $b_{22}$ as

$$
\begin{aligned}
& \langle\mathbf{i}\rangle=b_{11} \mathbf{E}+b_{12} \mathbf{g}, \\
& \langle\mathbf{j}\rangle=b_{21} \mathbf{E}+b_{22} \mathbf{g},
\end{aligned}
$$

with

$$
b_{11}=\Lambda
$$




$$
\begin{aligned}
& b_{12}=b_{21}=\varphi\left[\gamma_{\mathrm{p}}\left(\rho_{\mathrm{p}}-\rho\right)+\gamma_{\mathrm{c}}\left(\rho_{\mathrm{c}}-\rho\right)\right] \mu_{E}, \\
& b_{22}=\varphi\left[\gamma_{\mathrm{p}}\left(\rho_{\mathrm{p}}-\rho\right)+\gamma_{\mathrm{c}}\left(\rho_{\mathrm{c}}-\rho\right)\right] \frac{U}{g},
\end{aligned}
$$

where $\Lambda$ is the electric conductivity of the suspension. For charged composite spheres in a symmetric electrolyte, $\Lambda=$ $\Lambda^{\infty}+O(\bar{\sigma}, \bar{Q})$ with $\Lambda^{\infty}=\left(D_{+}+D_{-}\right) z^{2} e^{2} n^{\infty} / k T, \mu_{\mathrm{E}}$ is given by Eq. [59] to the second orders of $\bar{\sigma}$ and $\bar{Q}$, and $U$ can be evaluated using Eq. [45] to the second order.

\section{RESULTS AND DISCUSSION}

In this section, we first consider several limiting cases of the expressions [45] and [59] for the sedimentation velocity and electrophoretic mobility (or sedimentation potential), respectively, of charged composite spheres. The correctness of these expressions may be confirmed by examining some of these limiting cases for which analytical solutions are already known. Numerical results of the general cases are then presented.

When there is no permeable layer on the surface of the rigid particle core, one has $d=0, r_{0}=a, \gamma_{\mathrm{p}}=0, \gamma_{\mathrm{c}}=1$, $Q=0$, and $\lambda=0$. Equations [44a], [45], and [59] then reduce to

$$
\begin{aligned}
U_{00}= & \frac{V_{\mathrm{t}}\left(\rho_{\mathrm{c}}-\rho\right) g}{6 \pi \eta a}, \\
U= & U_{00}\left[1-\frac{\epsilon \bar{\sigma}^{2}}{96 \pi \eta}\left(\frac{1}{D_{+}}+\frac{1}{D_{-}}\right)\left(\frac{k T}{z e}\right)^{2}\right. \\
& \left.\times\left(\frac{\kappa a}{\kappa a+1}\right)^{2} \Theta_{1}(\kappa a, 1)+O\left(\bar{\sigma}^{3}\right)\right], \\
\mu_{\mathrm{E}}= & \frac{a \sigma}{\eta}\left(\frac{1}{\kappa a+1}\right) \Theta_{2}(\kappa a, 1)+O\left(\bar{\sigma}^{3}\right),
\end{aligned}
$$

where $\Theta_{1}$ and $\Theta_{2}$ are functions defined by

$$
\begin{aligned}
\Theta_{1}(x, y)= & y^{3} e^{2 x}\left[5 E_{6}(x)-3 E_{4}(x)\right]^{2} \\
& -8 e^{x}\left[E_{5}(x)-E_{3}(x)\right]+e^{2 x}\left[7 E_{8}(2 x)\right. \\
& \left.-3 E_{4}(2 x)-4 E_{3}(2 x)\right], \\
\Theta_{2}(x, y)= & \frac{2}{3}+\frac{1}{3} y^{3}-y^{3} e^{x}\left[5 E_{7}(x)-2 E_{5}(x)\right],
\end{aligned}
$$

and

$$
E_{n}(x)=\int_{1}^{\infty} t^{-n} e^{-x t} d t .
$$

Equation [63a] is the result of Stokes law. From Eq. [26] by letting $r_{0}=a$, one can obtain the following relation between the surface potential and the surface charge density of the rigid sphere at equilibrium:

$$
\sigma=\frac{\epsilon(\kappa a+1)}{4 \pi a} \psi^{(e q)}(a) .
$$

Substituting the above equation into Eqs. [63b] and [63c], these degenerated results are the same as those of a rigid sphere with a low surface potential (7). Note that, for the two extreme cases $\kappa a \rightarrow 0$ and $\kappa a \rightarrow \infty$, Eq. [63b] precisely reduces to $U=U_{00}$ and the sedimentation velocity limits to the Stokes velocity. Also, Eq. [63c] together with Eq. [66] leads to $\mu_{E}=\epsilon \psi^{(e q)}(a) / 6 \pi \eta$ as $\kappa a \rightarrow 0$ (the Hückel limit) and $\mu_{\mathrm{E}}=\epsilon \psi^{(e q)}(a) / 4 \pi \eta$ as $\kappa a \rightarrow \infty$ (the Smoluchowski limit).

When the composite particle is a homogeneous porous sphere, one has $r_{0}=0, d=a, \gamma_{\mathrm{p}}=1, \gamma_{\mathrm{c}}=0$, and $\sigma=0$. In this limiting case, Eqs. [44a], [45], and [59] become

$$
\begin{aligned}
U_{00}= & \frac{V_{\mathrm{t}}\left(\rho_{\mathrm{p}}-\rho\right) g}{6 \pi \eta a}\left[\frac{2(\lambda a)^{3} \cosh (\lambda a)+3 \alpha_{1}(\lambda a)}{2(\lambda a)^{2} \alpha_{1}(\lambda a)}\right], \\
U= & U_{00}+\frac{U_{00} \bar{Q}^{2}}{3(\lambda a)^{2} \alpha_{1}(\lambda a)}\left\{3 \alpha_{1}(\lambda a) \int_{0}^{a}\left(\frac{r}{a}\right)^{3} G_{20}(r) d r\right. \\
& +6 \int_{0}^{a} \alpha_{1}(\lambda r) G_{20}(r) d r+\left[2(\lambda a)^{3} \cosh (\lambda a)\right. \\
& \left.+3 \alpha_{1}(\lambda a)\right] \int_{a}^{\infty}\left(\frac{r}{a}\right)^{3} G_{20}(r) d r-3(\lambda a)^{2} \alpha_{1}(\lambda a) \\
& \times \int_{a}^{\infty}\left(\frac{r}{a}\right)^{2} G_{20}(r) d r+\left[\left(\lambda^{2} a^{2}+6\right) \alpha_{1}(\lambda a)\right. \\
& \left.\left.-2(\lambda a)^{2} \sinh (\lambda a)\right] \int_{a}^{\infty} G_{20}(r) d r\right\}+O\left(\bar{Q}^{3}\right) \\
\mu_{\mathrm{E}}= & \frac{Q}{\eta \lambda^{2}}\left\{1+\frac{1}{3}\left(\frac{\lambda}{\kappa}\right)^{2}\left(1+e^{-2 \kappa a}-\frac{1-e^{-2 \kappa a}}{\kappa a}\right)\right. \\
& +\frac{1}{3}\left(\frac{\lambda^{2}}{\lambda^{2}-\kappa^{2}}\right)\left(1+\frac{1}{\kappa a}\right) \\
& \times\left[\left(\frac{\lambda}{\kappa}\right)^{2} \frac{\kappa a\left(1+e^{-2 \kappa a}\right)-1+e^{-2 \kappa a}}{\lambda a \operatorname{coth}(\lambda a)-1}\right. \\
& \left.-1+e^{-2 \kappa a}\right]+O\left(\bar{Q}^{3}\right),
\end{aligned}
$$

where functions $G_{20}(r)$ and $\alpha_{1}(x)$ are defined by Eqs. [41c] and [A5a]. Equations [67b] and [67c] are identical to the formulas for the sedimentation velocity (27) and the electrophoretic mobility $(24,27,28)$, respectively, of a charged porous sphere derived previously.

When $\lambda \rightarrow \infty$ (very high segment density in the surface layer of the composite particle), the resistance to the fluid 
motion inside the surface layer is infinitely large. For this limiting case, Eqs. [44a], [45], and [59] become

$$
\begin{aligned}
U_{00}= & \frac{V_{\mathrm{t}}\left[\gamma_{\mathrm{p}}\left(\rho_{\mathrm{p}}-\rho\right)+\gamma_{\mathrm{c}}\left(\rho_{\mathrm{c}}-\rho\right)\right] g}{6 \pi \eta a} \\
U= & U_{00}\left\{1-\frac{\epsilon}{96 \pi \eta}\left(\frac{1}{D_{+}}+\frac{1}{D_{-}}\right)\left[\psi^{(e q)}(a)\right]^{2}\right. \\
& \left.\times \Theta_{1}\left(\kappa a, \frac{r_{0}}{a}\right)+O\left(\bar{\sigma}^{3}, \bar{\sigma}^{2} \bar{Q}, \bar{\sigma} \bar{Q}^{2}, \bar{Q}^{3}\right)\right\} \\
\mu_{\mathrm{E}}= & \frac{\epsilon \psi^{(e q)}(a)}{4 \pi \eta} \Theta_{2}\left(\kappa a, \frac{r_{0}}{a}\right)+O\left(\bar{\sigma}^{3}, \bar{\sigma}^{2} \bar{Q}, \bar{\sigma} \bar{Q}^{2}, \bar{Q}^{3}\right)
\end{aligned}
$$

[68c]

Note that in this case the mobile ions can still penetrate the surface layer and the fixed charges are distributed not only at the rigid core surface but also in the surface layer of the composite particle. Thus, the equilibrium potential distribution $\psi^{(e q)}(r)$, which is given by Eq. [26], and the results for the sedimentation velocity and sedimentation potential (or electrophoretic mobility) are generally different from those in the case of the true nonporous spheres of radius $a$.

When $\lambda \rightarrow 0$ (very low segment density), the surface layer of the composite particle does not exert any resistance to the fluid motion. Then, the function $F_{00 r}(r)$ in Eq. [30a] for the $r$ component of the fluid velocity reduces to

$$
F_{00 r}(r)=-\frac{1}{2}\left(\frac{r_{0}}{r}\right)^{3}+\frac{3}{2}\left(\frac{r_{0}}{r}\right)-1,
$$

and Eqs. [44a], [45], and [59] become

$$
\begin{aligned}
U_{00}= & \frac{V_{\mathrm{t}}\left[\gamma_{\mathrm{p}}\left(\rho_{\mathrm{p}}-\rho\right)+\gamma_{\mathrm{c}}\left(\rho_{\mathrm{c}}-\rho\right)\right] g}{6 \pi \eta r_{0}} \\
U= & U_{00}\left\{1-\frac{2 a}{3 r_{0}} \int_{r_{0}}^{\infty}\left(\frac{r}{a}\right)^{3} F_{00 r}(r)\left[G_{02}(r) \bar{\sigma}^{2}\right.\right. \\
& \left.+G_{11}(r) \bar{\sigma} \bar{Q}+G_{20}(r) \bar{Q}^{2}\right] d r \\
& \left.+O\left(\bar{\sigma}^{3}, \bar{\sigma}^{2} \bar{Q}, \bar{\sigma} \bar{Q}^{2}, \bar{Q}^{3}\right)\right\} \\
\mu_{\mathrm{E}}= & \frac{z e}{9 r_{0} \eta k T} \int_{r_{0}}^{\infty}\left(2 r^{3}+r_{0}^{3}\right) F_{00 r}(r) \\
& \times\left(\kappa \sigma \frac{d \psi_{e q 01}}{d r}+Q \frac{d \psi_{e q 10}}{d r}\right) d r \\
& +O\left(\bar{\sigma}^{3}, \bar{\sigma}^{2} \bar{Q}, \bar{\sigma} \bar{Q}^{2}, \bar{Q}^{3}\right)
\end{aligned}
$$

Here, functions $G_{02}(r), G_{11}(r)$, and $G_{20}(r)$ are defined by
Eq. [41] and functions $\psi_{e q 01}(r)$ and $\psi_{e q 10}(r)$ are given by Eq. [28].

To use the general expressions [44a], [45], and [59] for the sedimentation velocity and electrophoretic mobility and their simplified formulas [63], [67], [68], and [70] in the limiting cases, the parameters $\kappa a, \lambda a, r_{0} / a, \bar{\sigma}$, and $\bar{Q}$ of the colloidal system have to be determined. Experimental data for the surface layers of human erythrocytes (21) and rat lymphocytes (22) in electrolyte solutions indicated that the shielding length $1 / \lambda$ has values about $3 \mathrm{~nm}$ and that the magnitude of $Q$ ranges from quite low to about $1.6 \times 10^{6}$ $\mathrm{C} / \mathrm{m}^{3}$, depending on the $\mathrm{pH}$ value and ionic strength of the electrolyte solution. For some temperature-sensitive poly $(\mathrm{N}$ isopropylacrylamide) hydrogel layers on latex particles in salt solutions (23), values of $1 / \lambda$ were found to be about $1-50 \mathrm{~nm}$ and the magnitude of $Q$ could be as high as 8.7 $\times 10^{6} \mathrm{C} / \mathrm{m}^{3}$. As to the surface charge density, experimental study for the adsorption of poly(vinyl alcohol) onto AgI reported that the value of $\sigma$ changes from 0 to $-0.035 \mathrm{C} /$ $\mathrm{m}^{2}$ upon increasing the pAg from 5.6 to 11 , while experimental data for a positively charged polystyrene latex used as the adsorbent for the polyelectrolyte poly (acrylic acid) showed that $\sigma$ can have a value as high as $0.16 \mathrm{C} / \mathrm{m}^{2}(26)$. It is widely understood that the Debye length $1 / \kappa$ is in the range from angstroms to about a micron, depending on the ionic strength of the solution. For a composite particle with $\sigma=2 \times 10^{-3} \mathrm{C} / \mathrm{m}^{2}$ and $Q=2 \times 10^{6} \mathrm{C} / \mathrm{m}^{3}$ in an aqueous solution of a univalent electrolyte with $1 / \kappa=10^{-9} \mathrm{~m}$, one obtains the dimensionless charge densities $\bar{\sigma} \approx 0.1$ and $\bar{Q}$ $\approx 0.1$.

According to Eqs. [45] and [46], the sedimentation velocity of a charged composite sphere in a given electrolyte solution can be calculated to the second orders $\sigma^{2}, \sigma Q$, and $Q^{2}$. The corrections for the effects of the fixed distributed charges to $U$ start from $\sigma^{2}, \sigma Q$, and $Q^{2}$, instead of $\sigma$ and $Q$. The reason is that these effects are due to the interaction between the particle charges and the local induced sedimentation field; both are of orders $\sigma$ and $Q$, and thus the corrections are of orders $\sigma^{2}, \sigma Q$, and $Q^{2}$. Figures 2-5 show plots of numerical results for the dimensionless coefficients $H_{1}$, $H_{2}$, and $H_{3}$ in Eq. [46] as functions of parameters $r_{0} / a, \kappa a$, and $\lambda a$ for the sedimentation velocity of a charged composite sphere in an aqueous solution of $\mathrm{KCl}$ at room temperature. The values $\epsilon k^{2} T^{2} / 4 \pi \eta D_{+} z^{2} e^{2}=0.263$ and $\epsilon k^{2} T^{2} /$ $4 \pi \eta D_{-} z^{2} e^{2}=0.254$ (29) are used in the calculations. It is found from the numerical results that $H_{1}, H_{2}$, and $H_{3}$ are always positive values and satisfy the relation $H_{2}^{2} \leq 4 H_{1} H_{3}$. With this relation of inequality, Eq. [45] illustrates that the presence of the particle charges would reduce the magnitude of the sedimentation velocity.

Figure 2 illustrates that, for a specified value of the shielding ratio $\lambda a$ for a charged composite sphere with $r_{0} / a$ $=0.5$, the effects of the particle charges on the sedimentation velocity are maximal at $\kappa a$ equal to about $0.2-0.6$ and fade 

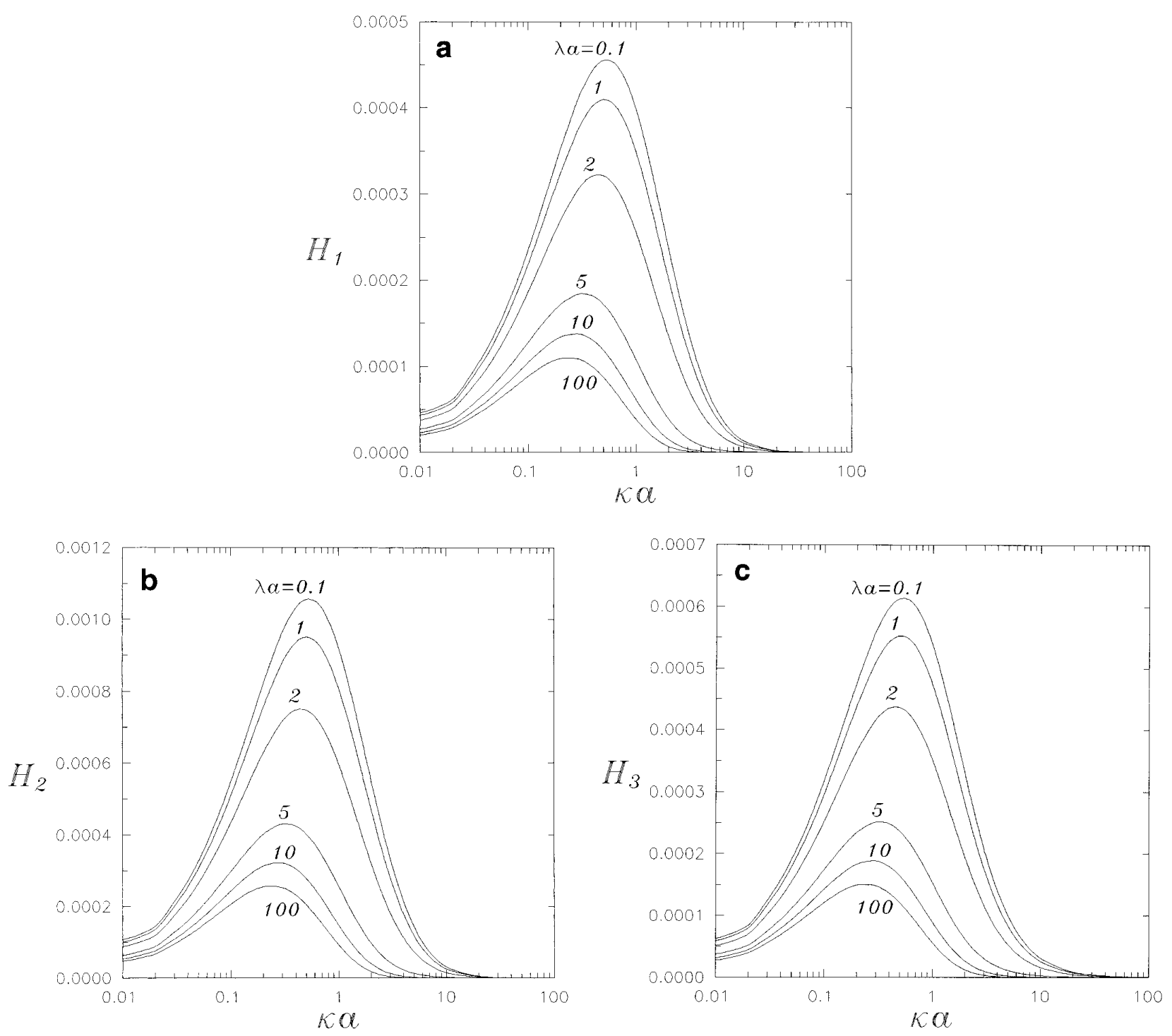

FIG. 2. Plot of the dimensionless coefficients $H_{1}, H_{2}$, and $H_{3}$ in Eq. [45] for a sedimenting composite sphere of $r_{0} / a=0.5$ versus $\kappa a$ at fixed values of $\lambda a$.

out when the value of $\kappa a$ is getting small or large. The reason for this behavior is obvious. The limit $\kappa a \rightarrow 0$ means that the effect of the presence of the counterions around the charged particle is negligible (viz. the particle is not affected by the electrostatic interaction with the surrounding counterions in the hydrodynamic behavior), while the limit $\kappa a \rightarrow \infty$ indicates that the total charge density is zero everywhere and the total electric force on the particle vanishes. Both limits result in $H_{1}=H_{2}=H_{3}=0$ (as they are calculated from Eqs. [46] and [44d,e, f ]) and the same sedimentation velocity as that of an uncharged composite particle, regardless of the values of $\lambda a$ and $r_{0} / a$. Note that the locations of the maxima in $H_{1}, H_{2}$, and $H_{3}$ shift to smaller $\kappa a$ as $\lambda a$ increases.

Figures 2-4 also show that $H_{1}, H_{2}$, and $H_{3}$ increase monotonically with decreasing $\lambda a$ for given values of $\kappa a$ and $r_{0} /$ $a$. When $\lambda a \rightarrow \infty$, the resistance to the fluid motion inside the surface layer of the composite particle is infinitely large and the velocity profile in the surface layer disappears. The ions can still penetrate the surface layer and the equilibrium potential distribution $\psi^{(e q)}$ is the same as Eq. [26]. Therefore, for given values of $\kappa a$ and $r_{0} / a$ with $\lambda a \rightarrow \infty, H_{1}, H_{2}$, and $H_{3}$ approach constant values as shown in Fig. 3 and in Eq. [68b]. When $\lambda a \rightarrow 0$, the surface layer does not exert resistance to the fluid motion, and $H_{1}, H_{2}$, and $H_{3}$ for given values of $\kappa a$ and $r_{0} / a$ approach another constant values in Fig. 3 given by Eq. [70b]. Note that these dimensionless coefficients are very sensitive functions of $\lambda a$ over the range of $\lambda a$ equal to $1-10$.

In Figs. 4 and 5, the coefficients $H_{1}, H_{2}$, and $H_{3}$ are plotted as functions of the parameter $r_{0} / a$ for given values of $\kappa a$ and $\lambda a$. It can be seen in Figs. $4 \mathrm{a}$ and $5 \mathrm{a}$ that $H_{1}$ is a 

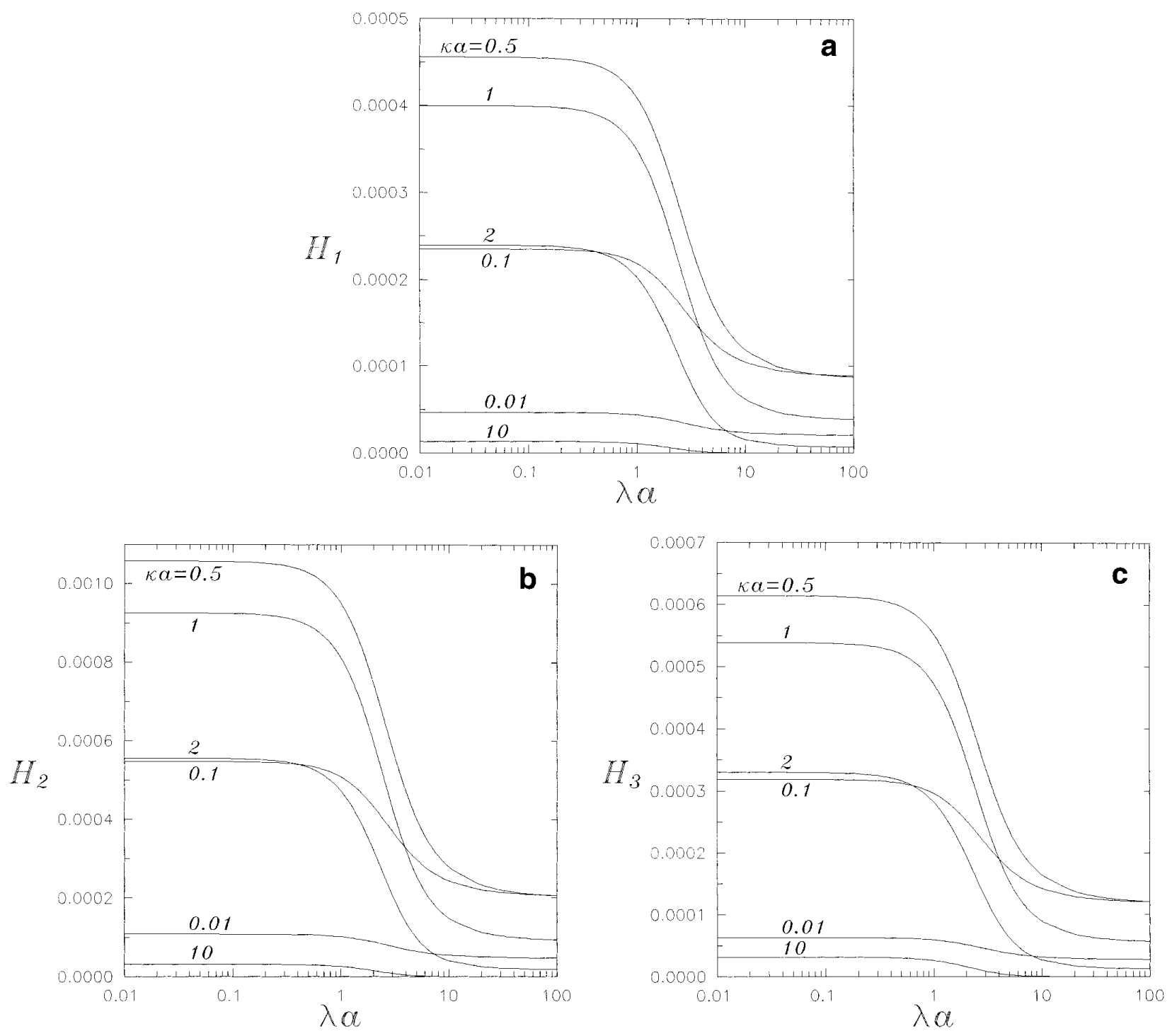

FIG. 3. Plot of the coefficients $H_{1}, H_{2}$, and $H_{3}$ in Eq. [45] for a sedimenting composite sphere of $r_{0} / a=0.5$ versus $\lambda a$ at fixed values of $\kappa a$.

monotonic increasing function of $r_{0} / a$ for fixed values of $\kappa a$ and $\lambda a$. In the special case of $r_{0} / a=0$, the composite particle degenerates to a homogeneous porous sphere and $H_{1}$ must be equal to zero. Obviously, $H_{1}$ is independent of $\lambda a$ in the special case of $r_{0} / a=1$ in which the composite particle reduces to a rigid sphere with no surface layer. Conversely, as illustrated in Figs. $4 \mathrm{c}$ and $5 \mathrm{c}, H_{3}$ decreases monotonically with increasing $r_{0} / a$ and vanishes when $r_{0} / a=1$. On the other hand, $H_{2}$ equals zero in both limiting cases of $r_{0} / a=$ 0 and $r_{0} / a=1$ and there exists a maximal value of $H_{2}$ for given values of $\kappa a$ and $\lambda a$, as shown in Figs. $4 \mathrm{~b}$ and $5 \mathrm{~b}$.

The dimensionless coefficients $H_{\sigma}$ and $H_{Q}$ for the sedimentation potential or electrophoretic mobility in the $\mathrm{KCl}$ solution calculated from Eq. [60] are plotted as functions of parameters $\kappa a, \lambda a$, and $r_{0} / a$ in Figs. 6-9. It can be seen that these dimensionless coefficients, which are always positive, are monotonic decreasing functions of $\kappa a$ for given values of $\lambda a$ and $r_{0} / a$ and are also monotonic decreasing functions of $\lambda a$ for fixed values of $\kappa a$ and $r_{0} / a$. When $\kappa a$ is small ( $\lesssim 5$ ), $H_{\sigma}$ and $H_{Q}$ have the same order of magnitude; however, when $\kappa a$ is large $(\gtrsim 20)$, the magnitude of $H_{\sigma}$ is much smaller than that of $H_{Q}$. Figure 6 illustrates that $H_{\sigma}$ is proportional to $(\kappa a)^{-1}$ and $H_{Q}$ is independent of $\kappa a$ when $\kappa a \gg 1$, consistent with the predictions obtained by assuming the particle surface is planar (19), and that both $H_{\sigma}$ and $H_{Q}$ are not sensitive functions of $\kappa a$ when $\kappa a \ll 1$, similar to the relevant results for charged solid spheres (7) and charged porous spheres (27). Figure 7 indicates that both $H_{\sigma}$ and $H_{Q}$ are independent of $\lambda a$ when $\lambda a \gg 1$ or $\lambda a \ll 1$, in accordance with Eqs. [68c] and [70c]. The value of $H_{\sigma}$ for large $\lambda a$ for case of $\kappa a=100$ is very small and cannot be shown in the range of the logarithmic plot of Fig. 7a. As exhibited in Figs. 8a and 9a, $H_{\sigma}$ is a monotonic increasing function of $r_{0} / a$ for fixed values of $\kappa a$ and $\lambda a$. In the limiting 

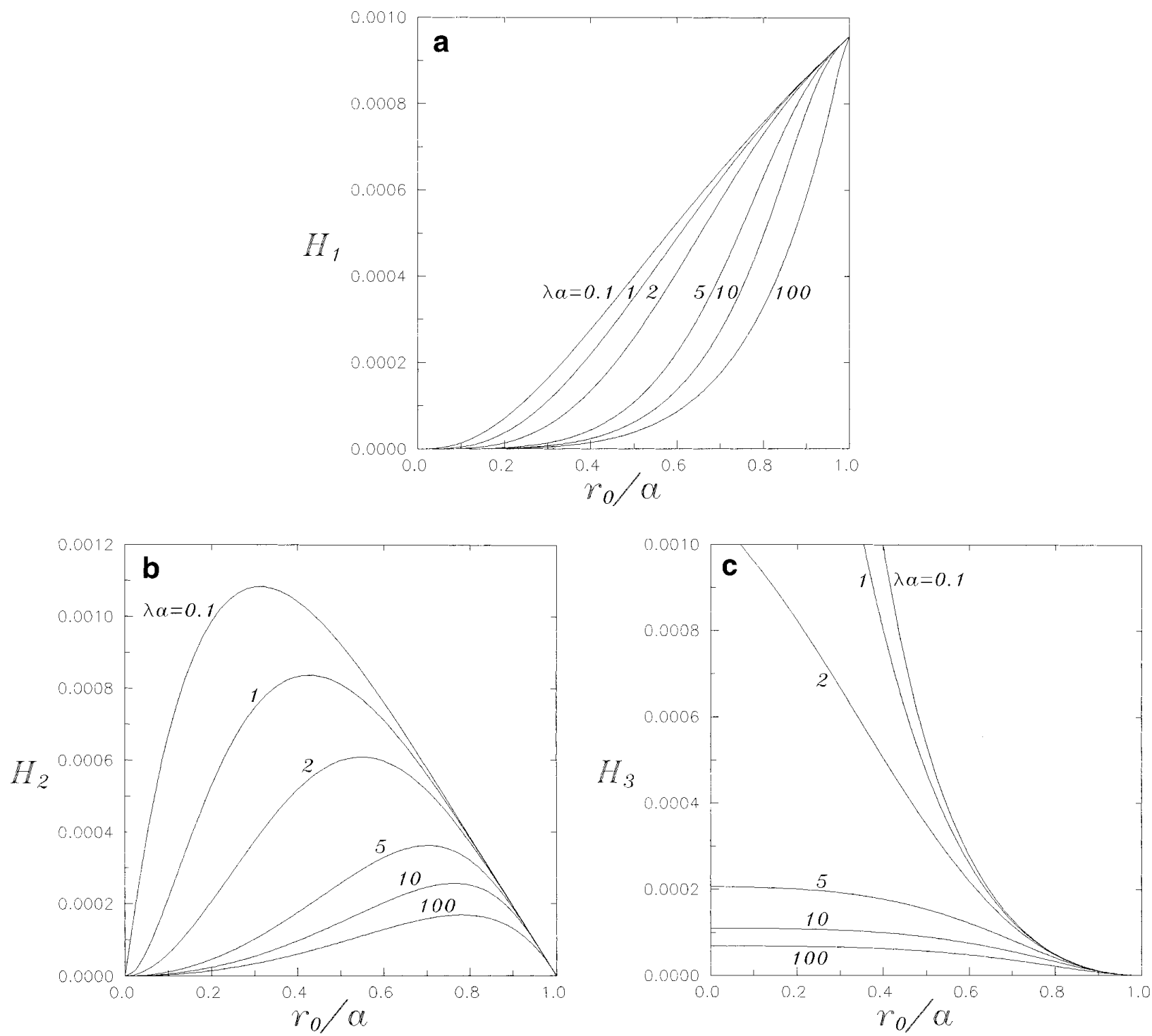

FIG. 4. Plot of the coefficients $H_{1}, H_{2}$, and $H_{3}$ in Eq. [45] for a sedimenting composite sphere with $\kappa a=1$ versus $r_{0} / a$ at fixed values of $\lambda a$.

case of $r_{0} / a=0, H_{\sigma}$ must equal nothing. However, as can be seen in Figs. $8 \mathrm{~b}$ and $9 \mathrm{~b}, H_{Q}$ decreases monotonically with increasing $r_{0} / a$ for given values of $\kappa a$ and $\lambda a$ and equals zero when $r_{0} / a=1$.

Theoretical studies $(30,31)$ have predicted that a nonuniformly charged but "neutral" impermeable sphere (with zero area-averaged zeta potential) can translate in electric fields. It would be of interest to know whether composite spheres with zero net charge $\left(4 \pi r_{0}^{2} \sigma+(4 \pi / 3)\left(a^{3}-\right.\right.$ $\left.r_{0}^{3}\right) Q=0$ ) can undergo electrophoresis or produce sedimentation potential, and be retarded by this potential gradient during the sedimentation. For such spherically-symmetric "neutral" composite particles (with $\bar{\sigma}=-(\kappa a / 3)\left[\left(a / r_{0}\right)^{2}\right.$ $\left.\left.-\left(r_{0} / a\right)\right] \bar{Q}\right)$, Eqs. [45] and [59] become

$$
U=U_{00}\left[1-(\kappa a)^{4} H_{0} \bar{Q}^{2}+O\left(\bar{Q}^{3}\right)\right], \quad[71 \mathrm{a}]
$$

$$
\mu_{\mathrm{E}}=H_{\mu} \frac{a^{2} Q}{\eta}+O\left(\bar{Q}^{3}\right),
$$

where

$$
\begin{aligned}
H_{0}= & H_{3}-\frac{1}{3}\left[\left(\frac{a}{r_{0}}\right)^{2}-\left(\frac{r_{0}}{a}\right)\right] H_{2} \\
& +\frac{1}{9}\left[\left(\frac{a}{r_{0}}\right)^{2}-\left(\frac{r_{0}}{a}\right)\right]^{2} H_{1}, \\
H_{\mu}= & H_{Q}-\frac{1}{3}\left[\left(\frac{a}{r_{0}}\right)^{2}-\left(\frac{r_{0}}{a}\right)\right] H_{\sigma} .
\end{aligned}
$$

Figure 10 displays the numerical results for the dimen- 

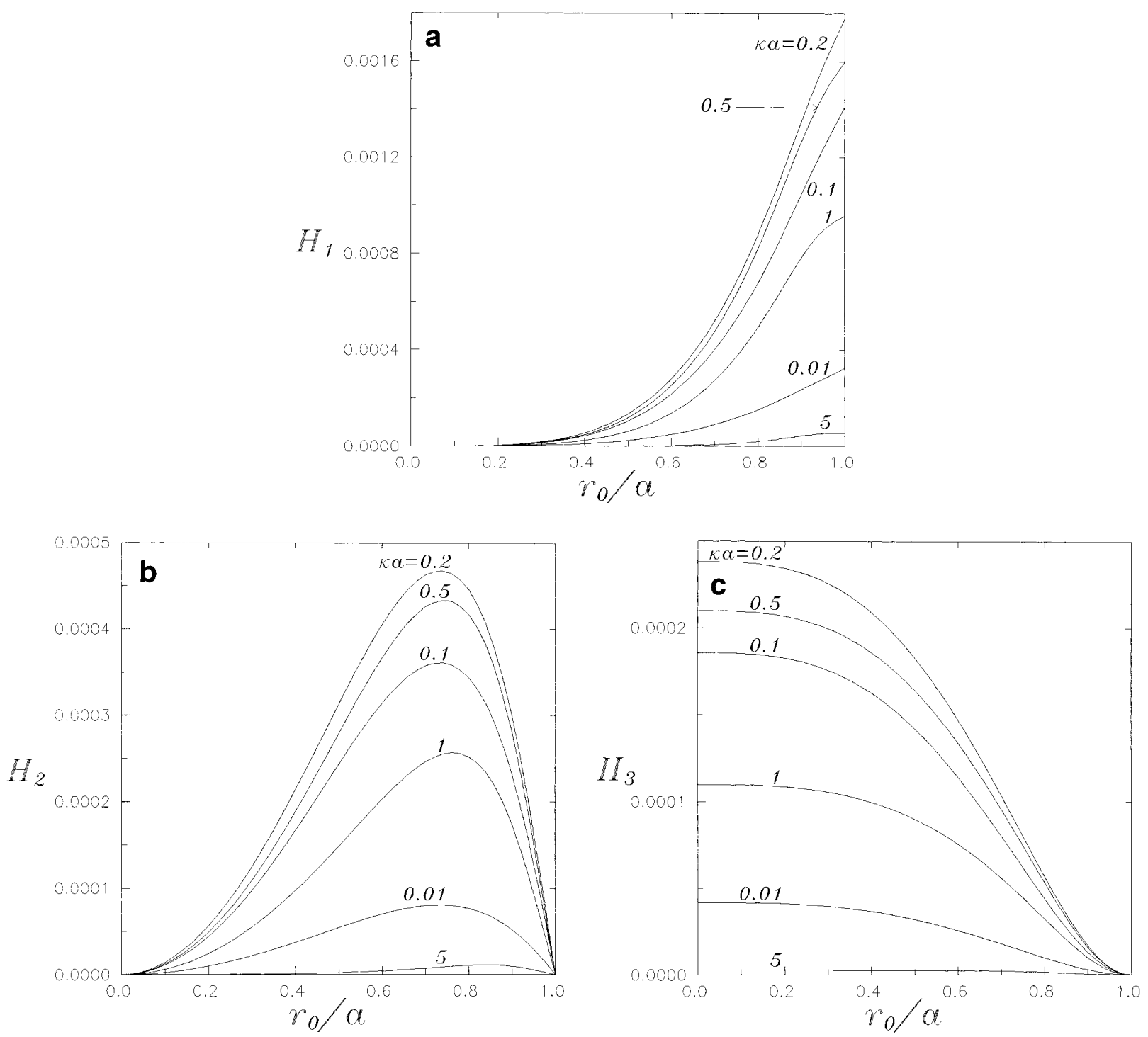

FIG. 5. Plot of the coefficients $H_{1}, H_{2}$, and $H_{3}$ in Eq. [45] for a sedimenting composite sphere with $\lambda a=10$ versus $r_{0} / a$ at fixed values of $\kappa a$.

sionless coefficient $H_{0}$ for the sedimentation velocity of a composite sphere in the $\mathrm{KCl}$ solution as functions of parameters $\kappa a, \lambda a$, and $r_{0} / a$. It can be seen that $H_{0}$ is positive and the presence of the fixed-charge distribution in the "neutral", particle would reduce its settling velocity. The tendency of the dependence of $H_{0}$ on $\kappa a, \lambda a$, and $r_{0} / a$ is quite similar to that of the coefficient $H_{3}$ presented in Figs. 2c-5c, except that the values of $H_{0}$ are about two orders of magnitude smaller and its maxima for fixed values of $\lambda a$ and $r_{0} / a$ occur at values of $\kappa a$ about an order of magnitude larger.

The dimensionless coefficient $H_{\mu}$ for the electrophoretic mobility or sedimentation potential associated with composite spheres in the $\mathrm{KCl}$ solution is drawn as a function of parameters $\kappa a, \lambda a$, and $r_{0} / a$ in Fig. 11. It can be seen that $H_{\mu}$ is always positive so that the "neutral" composite spheres can experience electrophoresis or generate sedimentation potential. The direction of the electrophoretic velocity or the induced potential gradient is decided by the fixed charges in the porous surface layers (rather than the surface charges of the rigid cores) of the particles. Similar to the coefficient $H_{Q}$ shown in Figs. $6 \mathrm{~b}$ and $8 \mathrm{~b}, H_{\mu}$ decreases monotonically with the increase of $\lambda a$ for given values of $\kappa a$ and $r_{0} / a$. However, $H_{\mu}$ is not necessarily a monotonic function of either $\kappa a$ (as $\lambda a$ is large or $r_{0} / a$ is small) or $r_{0} / a$ (as $\lambda a$ is small or $\kappa a$ is large). The trend of the dependence of $H_{\mu}$ on $\kappa a$ and $r_{0} / a$ is quite different from that of $H_{Q}$ presented in Figs. $6 \mathrm{~b}-9 \mathrm{~b}$. When $\kappa a$ is large $(\gtrsim 10)$, the coefficients $H_{\mu}$ and $H_{Q}$ have the same order of magnitude, but when $\kappa a$ is as small as 0.1 , the values of $H_{\mu}$ are about three orders of magnitude smaller than the values of $H_{Q}$.

\section{CONCLUDING REMARKS}

The steady-state electrokinetic phenomena of charged composite particles under the action of gravity in an un- 

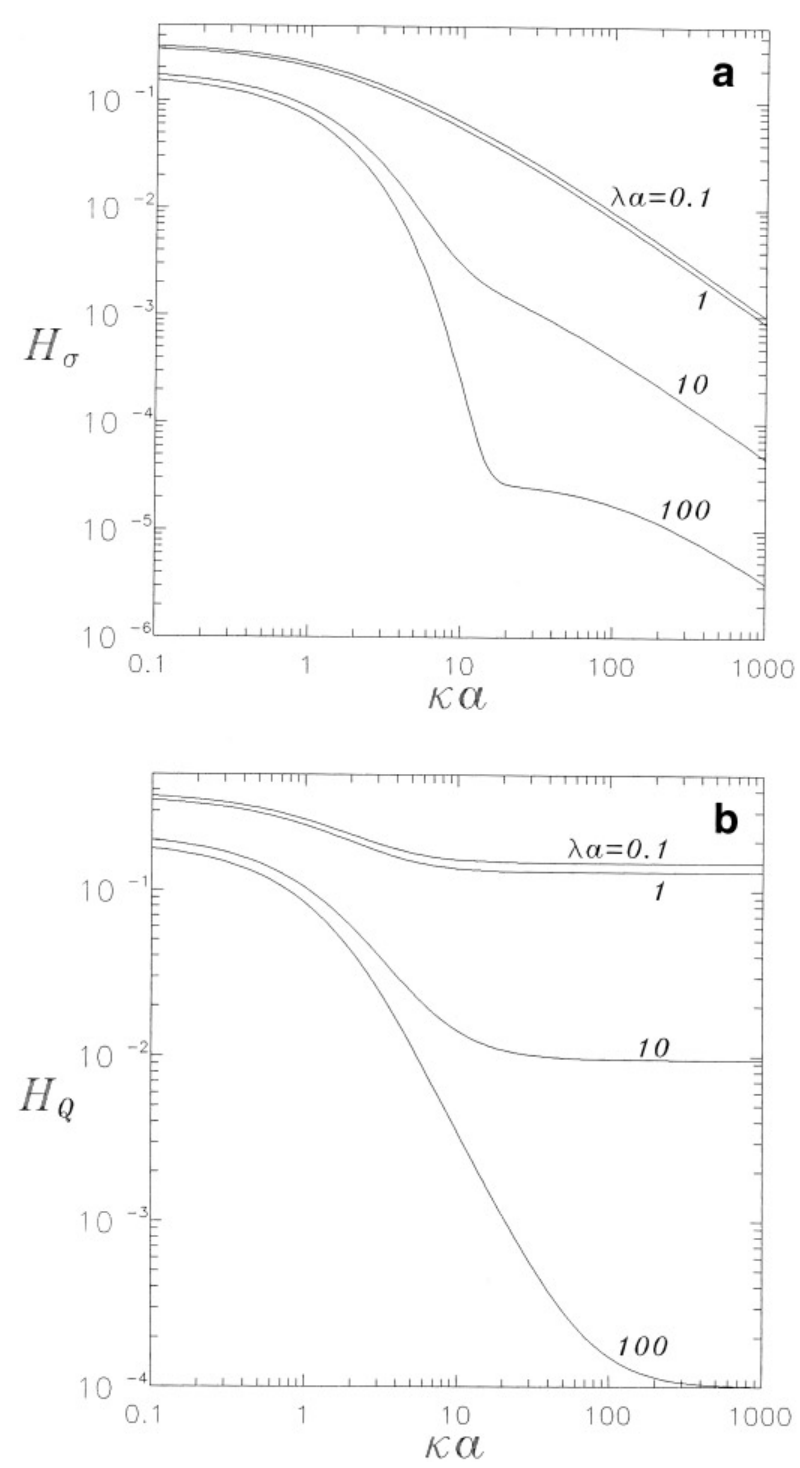

FIG. 6. Plot of the dimensionless coefficients $H_{\sigma}$ and $H_{Q}$ in Eq. [59] for a dilute suspension of identical composite spheres of $r_{0} / a=0.5$ versus $\kappa a$ at fixed values of $\lambda a$.

bounded electrolyte solution are analyzed in this work. The surface layer of each composite particle is treated as a solvent-permeable and ion-penetrable shell in which fixedcharged groups and frictional segments are distributed at uniform densities. Solving the linearized continuity equations of ions, Poisson-Boltzmann equation, and combined modified Stokes/Brinkman flow equations applicable to the system of an isolated composite sphere by a regular perturbation method, we have obtained the ion concentration distributions, the electric potential profile, and the fluid flow field. Since the electric potential distribution differs from the equilibrium values, an electric force acting on the charged composite particle is induced. The total force exerted on the particle is the sum of the gravitational, electric, and hydrodynamic forces, and the requirement that the total force is zero leads to an explicit formula, Eq. [45], for the settling velocity of the charged composite sphere. The corrections for the effects of the fixed distributed charges to the settling velocity begin at the second order $\left(\sigma^{2}, \sigma Q\right.$, and $\left.Q^{2}\right)$. Numerical results indicate that these effects have a maximum at a finite value of $\kappa a$ (equal to about $0.2-0.6$ as $r_{0} / a=0.5$ ) and disappear when $\kappa a$ approaches zero and infinite. The analytical expression for the average electric current density in a dilute suspension of identical composite spheres is given by Eq. [56]. The explicit formula, Eq. [58], for the sedimentation potential is derived from Eq. [56] by letting the net current in the suspension be zero. The Onsager reciprocal relation is satisfied between the sedimentation potential and the electrophoretic mobility. It is also found that the firstorder dimensionless coefficients $\left(H_{\sigma}\right.$ and $\left.H_{Q}\right)$ for the sedi-
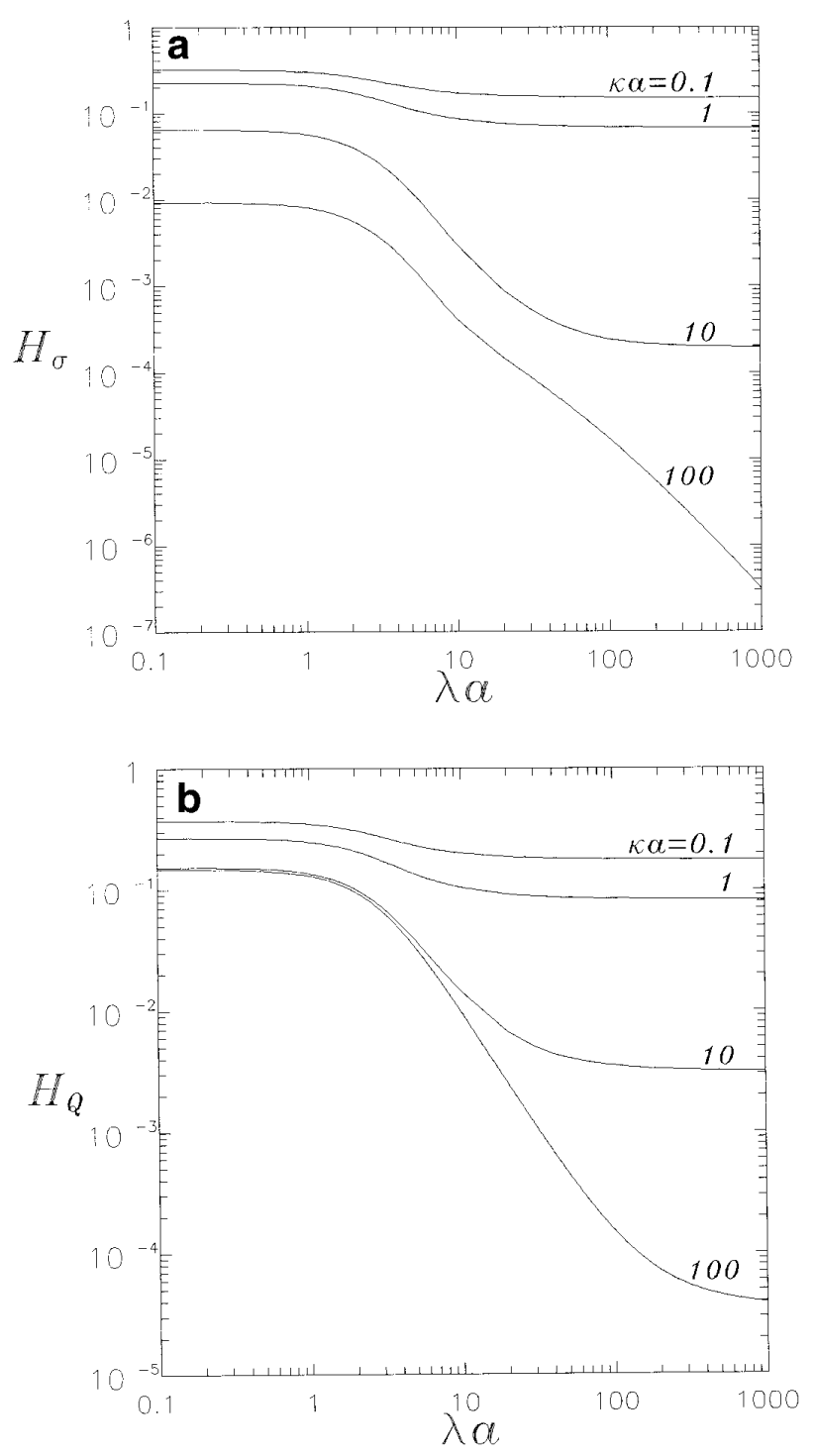

FIG. 7. Plot of the coefficients $H_{\sigma}$ and $H_{Q}$ in Eq. [59] for a dilute suspension of identical composite spheres of $r_{0} / a=0.5$ versus $\lambda a$ at fixed values of $\kappa a$. 

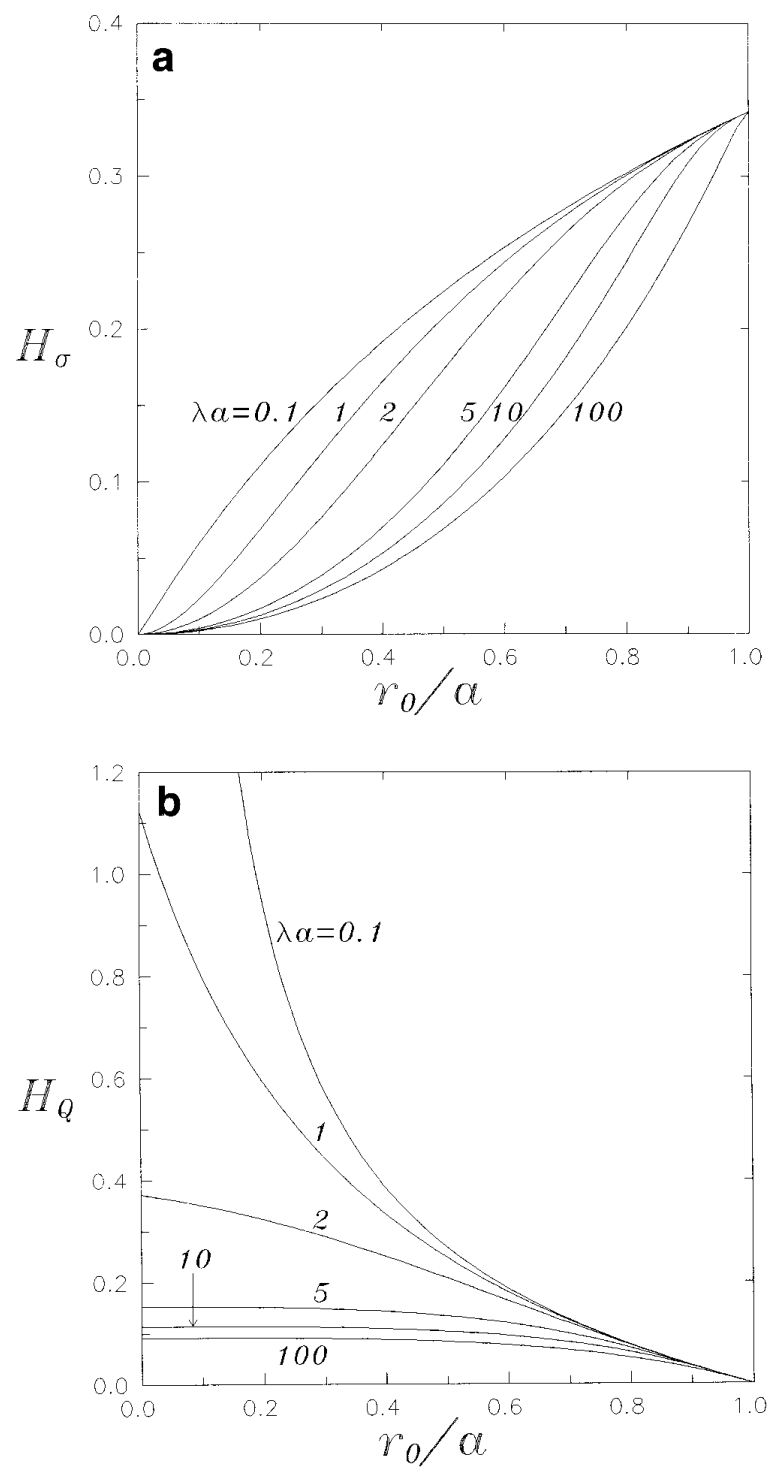

FIG. 8. Plot of the coefficients $H_{\sigma}$ and $H_{Q}$ in Eq. [59] for a dilute suspension of identical composite spheres with $\kappa a=1$ versus $r_{0} / a$ at fixed values of $\lambda a$.

mentation potential or electrophoretic mobility are monotonic decreasing functions of $\kappa a$ for constant values of $\lambda a$ and $r_{0} / a$ and that they are also monotonic decreasing functions of $\lambda a$ for fixed values of $\kappa a$ and $r_{0} / a$. Expressions [45] and [58] for the sedimentation velocity and sedimentation potential (or electrophoretic mobility) in a dilute suspension of charged composite spheres reduce to the corresponding formulas for the charged solid spheres and the charged porous spheres, respectively, in the limiting cases of $r_{0} / a=1$ and $r_{0} / a=0$.

It is worth repeating that the Onsager relation between sedimentation and electrophoresis holds not only for impermeable charged particles but also for porous or composite ones. Another electrokinetic phenomenon in a circular capillary with the solution-permeable surface charge layer under the Debye-Hückel approximation has been generally studied (32), and similarly the electroosmotic flow rate and the streaming potential were shown to satisfy the Onsager reciprocity principle whether the capillary wall adsorbs a surface charge layer or not. Hence, different electrokinetic processes could reflect the same intrinsic phenomena, and measurements of one type of process can be used to check those of another. For instance, one can first measure the sedimentation potential in a dilute suspension of particles and then predict the electrophoretic mobility of the particles in the same system without any other measurements.

Additionally, Eqs. [45] and [58] with Eqs. [44] and [59] are obtained on the basis of the Debye-Hückel approximation for the equilibrium potential distribution around a composite sphere. A similar formula for the sedimentation veloc-
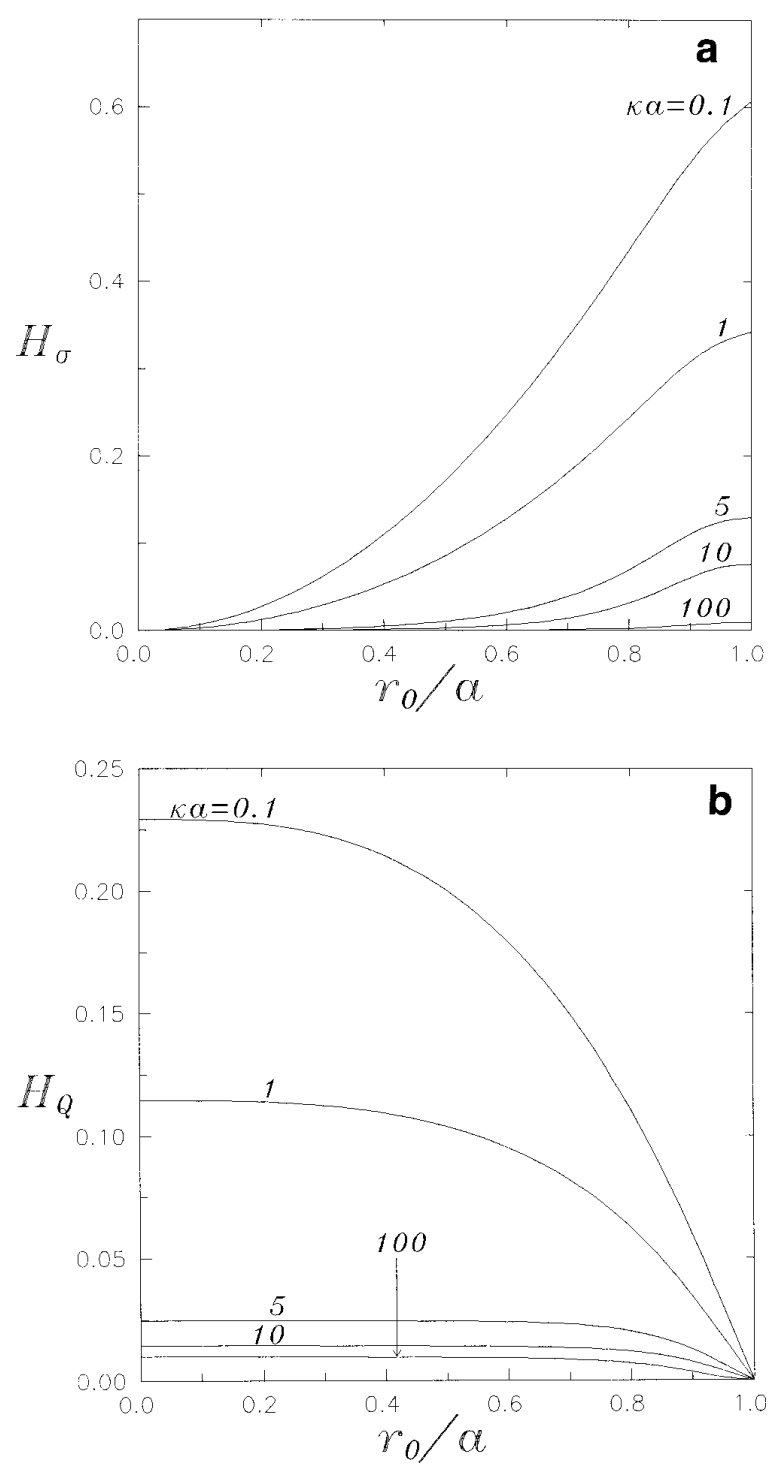

FIG. 9. Plot of the coefficients $H_{\sigma}$ and $H_{Q}$ in Eq. [59] for a dilute suspension of identical composite spheres with $\lambda a=10$ versus $r_{0} / a$ at fixed values of $\kappa a$. 
a

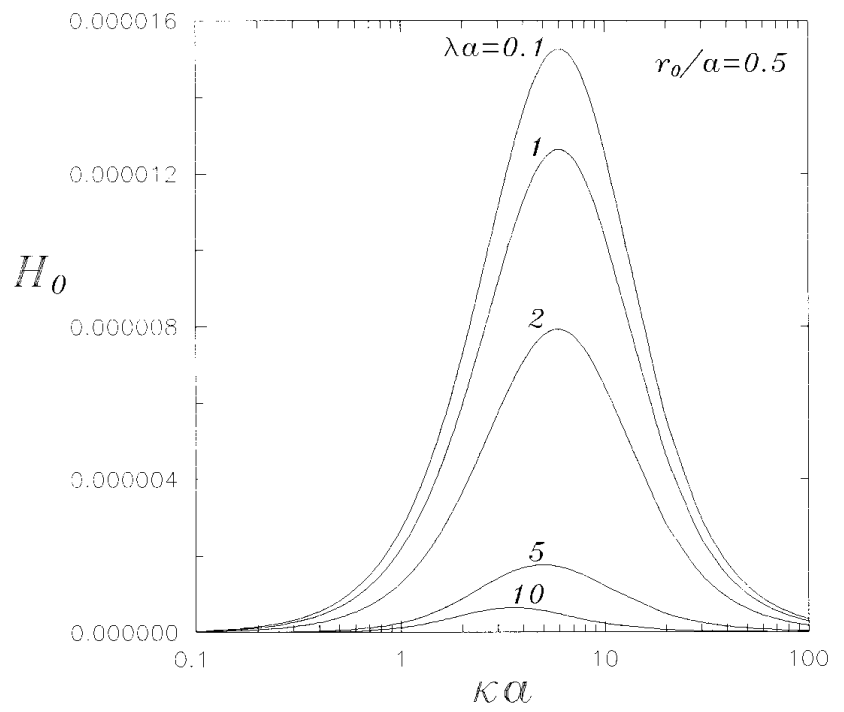

b

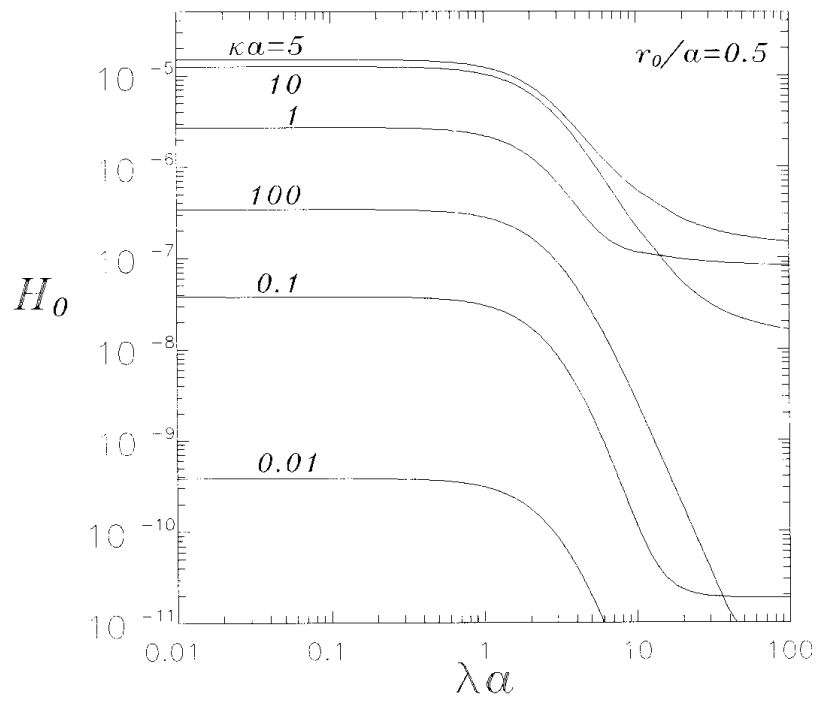

C

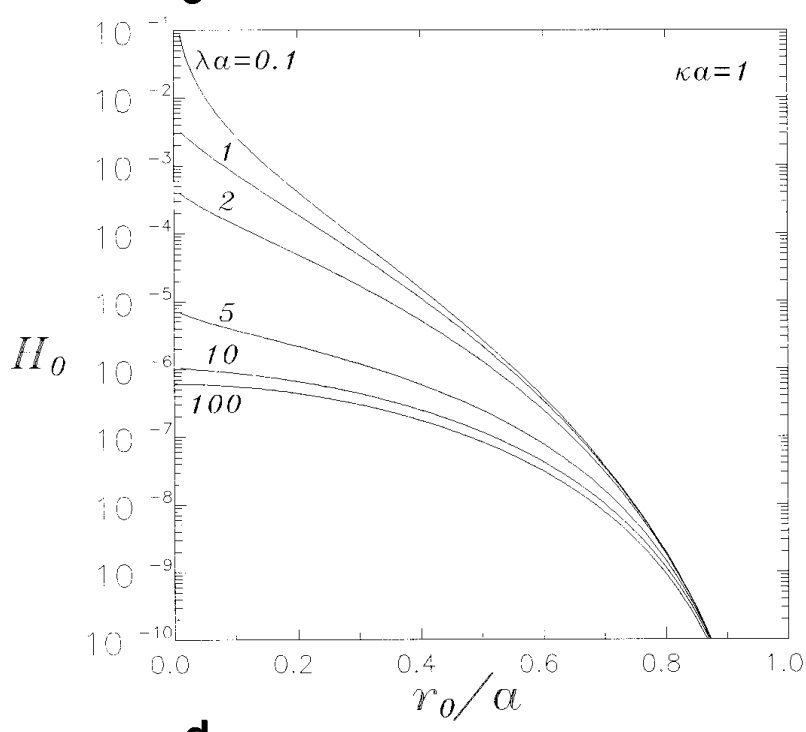

d

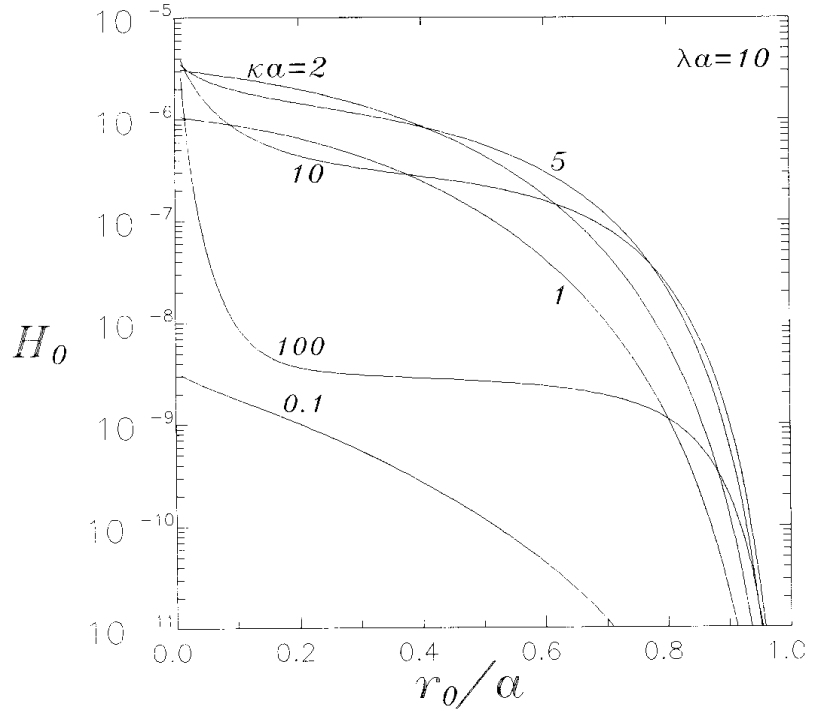

FIG. 10. Plot of the dimensionless coefficient $H_{0}$ in Eq. [71a] for a sedimenting composite sphere with zero net charge versus parameters $\kappa a$, $\lambda a$, and $r_{0} / a$.

ity of an impermeable rigid sphere with low $\zeta$ potential was shown to give an excellent approximation for the case of reasonably high $\zeta$ potential (with an error less than $0.1 \%$ for $\zeta_{e} / k T \leq 2$ in a $\mathrm{KCl}$ solution) $(7)$. Therefore, our results might be used tentatively for the situation of reasonably high electric potentials. On the other hand, the decay of the density distributions of frictional segments (33) and fixed charges (20) in the surface layer of the composite particle with the distance from the rigid core surface has not been considered in our calculations. In order to see whether our theory can be reasonably extended to the higher values of electric potential or to the nonuniform density distributions of frictional segments and fixed charges, we propose to obtain a numerical solution of the electrokinetic differential equations with no assumption on the magnitude of electric potential allowing the use of arbitrary distributions of fixed charge and fluid drag components in the composite particle and compare it with the approximate solution.

\section{APPENDIX}

\section{The Perturbation Equations and Solutions for the Motion of a Charged Composite Sphere in Symmetric E lectrolytes}

After the perturbation expansions given by Eq. [29] and the equilibrium distributions given by Eqs. [26] and [16] are substituted into Eqs. [7a], [8a], [9a], [11], and [17][22], and after like powers of $\bar{\sigma}$ and $\bar{Q}$ are equated on both sides of the respective equations, a set of differential equa- 

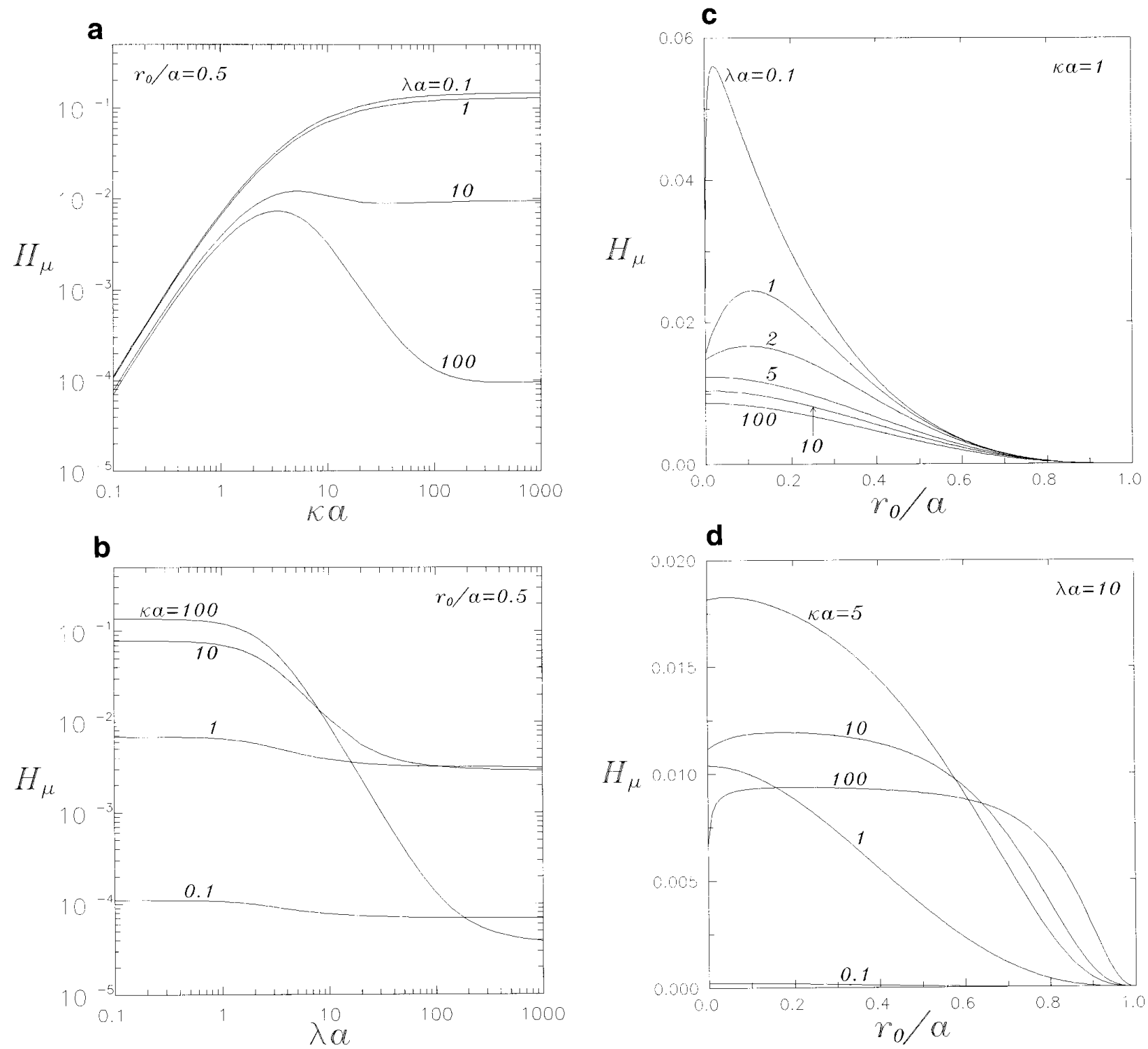

FIG. 11. Plot of the dimensionless coefficient $H_{\mu}$ in Eq. [71b] for a dilute suspension of identical composite spheres with zero net charge versus parameters $\kappa a, \lambda a$, and $r_{0} / a$.

tions and boundary conditions for each set of the functions $n_{i j \pm}, \mathbf{u}_{i j}, p_{i j}$, and $\psi_{i j}$ with $i$ and $j$ equal to 0,1 , and 2 can be obtained.

\section{Zeroth-Order Perturbations}

Collecting the zeroth-order terms in the procedure of this regular perturbation gives

$$
\begin{aligned}
\nabla^{2} \mathbf{u}_{00}-\mathrm{h}(r) \lambda^{2} \mathbf{u}_{00} & =\frac{1}{\eta} \nabla p_{00}, \\
\nabla \cdot \mathbf{u}_{00} & =0 \\
\left.\mathbf{u}_{00}\right|_{r=r_{0}} & =\mathbf{0}
\end{aligned}
$$

$$
\begin{gathered}
\left.\mathbf{u}_{00}\right|_{r=a^{+}}=\left.\mathbf{u}_{00}\right|_{r=a^{-}}, \\
\left.\mathbf{n} \cdot \boldsymbol{\sigma}_{00}^{H}\right|_{r=a^{+}}=\left.\mathbf{n} \cdot \boldsymbol{\sigma}_{00}^{H}\right|_{r=a^{-}}, \\
\left.\mathbf{u}_{00}\right|_{r \rightarrow \infty} \rightarrow-U_{00} \mathbf{e}_{z} .
\end{gathered}
$$

The solution for the $r$ and $\theta$ components of the velocity $\mathbf{u}_{00}$ and the pressure $p_{00}$ satisfying the above equations is

$$
\begin{aligned}
& u_{00 r}=U_{00} F_{00 r}(r) \cos \theta, \\
& u_{00 \theta}=U_{00} F_{00 \theta}(r) \sin \theta, \\
& p_{00}=\frac{\eta}{a} U_{00} F_{P 00}(r) \cos \theta,
\end{aligned}
$$


where

$$
\begin{aligned}
F_{00 r}(r)= & C_{001}+C_{002}\left(\frac{a}{r}\right)^{3}+C_{003}\left(\frac{a}{r}\right)^{3} \alpha_{1}(\lambda r) \\
& +C_{004}\left(\frac{a}{r}\right)^{3} \beta_{1}(\lambda r), \\
F_{00 \theta}(r)= & -C_{001}+\frac{C_{002}}{2}\left(\frac{a}{r}\right)^{3}-\frac{C_{003}}{2}\left(\frac{a}{r}\right)^{3} \alpha_{2}(\lambda r) \\
& -\frac{C_{004}}{2}\left(\frac{a}{r}\right)^{3} \beta_{2}(\lambda r), \\
F_{P 00}(r)= & \lambda^{2} a r\left[-C_{001}+\frac{C_{002}}{2}\left(\frac{a}{r}\right)^{3}\right], \\
& \text { if } r_{0}<\frac{r}{2} a ; \\
F_{00 r}(r)= & C_{005}\left(\frac{a}{r}\right)^{3}+C_{006} \frac{a}{r}-1, \\
F_{00 \theta}(r)= & \frac{C_{005}}{2}\left(\frac{a}{r}\right)^{3}-\frac{C_{006}}{2}\left(\frac{a}{r}\right)+1, \\
F_{P 00}(r)= & C_{006}\left(\frac{a}{r}\right)^{2}, \quad \text { if } a<r .
\end{aligned}
$$

In Eq. [A4],

$$
\alpha_{1}(x)=x \cosh x-\sinh x,
$$

$$
\alpha_{2}(x)=\left(x^{2}+1\right) \sinh x-x \cosh x,
$$

$$
\beta_{1}(x)=x \sinh x-\cosh x,
$$

$$
\beta_{2}(x)=\left(x^{2}+1\right) \cosh x-x \sinh x
$$

$C_{001}=\frac{3}{\Omega}\left[\lambda r_{0}-\lambda a \cosh (\lambda d)+\sinh (\lambda d)\right]$,

$$
C_{002}=\frac{\Gamma}{(\lambda a)^{3} \Omega \Delta}+2 C_{001}
$$

$$
C_{003}=\frac{-3}{(\lambda a)^{3} \Omega}\left\{A+3 \lambda r_{0}\left[\beta_{1}(\lambda a)-\beta_{1}\left(\lambda r_{0}\right)\right]\right\}
$$

$C_{004}=\frac{3}{(\lambda a)^{3} \Omega}\left\{\left[2(\lambda a)^{3}+\left(\lambda r_{0}\right)^{3}\right] \sinh \left(\lambda r_{0}\right)\right.$

$$
\left.+3 \lambda r_{0}\left[\alpha_{1}(\lambda a)-\alpha_{1}\left(\lambda r_{0}\right)\right]\right\}
$$

[A6d]

$$
C_{005}=1+C_{001}+C_{002}+\alpha_{1}(\lambda a) C_{003}
$$$$
+\beta_{1}(\lambda a) C_{004}-\frac{\Gamma}{2 \lambda a \Omega \Delta}
$$

[A6e ]

$$
C_{006}=\frac{\Gamma}{2 \lambda a \Omega \Delta}
$$
[A6f] where

$$
\begin{aligned}
\Delta= & \lambda r_{0} \sinh (\lambda a)-\cosh \left(\lambda r_{0}\right), \quad[\mathrm{A} 7 \mathrm{a}] \\
\Omega= & -6 \lambda r_{0}+\left[2(\lambda a)^{3}+\left(\lambda r_{0}\right)^{3}+3 \lambda a+3 \lambda r_{0}\right] \\
& \times \cosh (\lambda d)+\left[3\left(\lambda r_{0}\right)^{2}-3\right] \sinh (\lambda d), \quad[\mathrm{A} 7 \mathrm{~b}] \\
\Gamma= & 3 \lambda r_{0} A-9\left(\lambda r_{0}\right)^{2}\left[\beta_{1}(\lambda a)+\beta_{1}\left(\lambda r_{0}\right)\right] \\
& +3\left\{-\lambda a A+\lambda r_{0}\left[2(\lambda a)^{3}+\left(\lambda r_{0}\right)^{3}+3 \lambda r_{0}\right]\right. \\
& \left.\times \beta_{1}(\lambda a)+3 \lambda^{2} a r_{0} \beta_{1}\left(\lambda r_{0}\right)\right\} \cosh (\lambda d) \\
& +3\left\{A+3 \lambda r_{0}\left[\left(\lambda r_{0}\right)^{2} \beta_{1}(\lambda a)-\beta_{1}\left(\lambda r_{0}\right)\right]\right\} \sinh (\lambda d) .
\end{aligned}
$$

In Eq. [A7c],

$$
A=\left[2(\lambda a)^{3}+\left(\lambda r_{0}\right)^{3}\right] \cosh \left(\lambda r_{0}\right) .
$$

\section{First-Order Perturbations}

After collecting the $O\left(\bar{\sigma}^{j} \bar{Q}^{i}\right)$ terms with $(i, j)$ equal to $(0,1)$ and $(1,0)$ in the perturbation procedure, one obtains the following equations:

$$
\begin{gathered}
\nabla^{2} \mathbf{u}_{i j}-\mathrm{h}(r) \lambda^{2} \mathbf{u}_{i j}=\frac{1}{\eta} \nabla p_{i j}, \\
\nabla \cdot \mathbf{u}_{i j}=0, \\
\frac{z e n^{\infty}}{k T} \mathbf{u}_{00} \cdot \nabla \psi_{e q i j} \pm D_{ \pm} \nabla \cdot\left(\nabla n_{i j \pm} \pm \frac{z e n^{\infty}}{k T} \nabla \psi_{i j}\right)=0 \\
\nabla^{2} \psi_{i j}=-\frac{4 \pi z e}{\epsilon}\left(n_{i j+}-n_{i j-}\right) .
\end{gathered}
$$

[A6a] To obtain simpler forms of Eqs. [A10] and [A11], one can use the concept of the electrochemical potential and define

$$
\mu_{i j \pm}=\frac{k T}{z e n^{\infty}} n_{i j \pm} \pm \psi_{i j}
$$

Substituting Eqs. [A3] and [A12] into Eqs. [A10] and [A11], one obtains

$$
\begin{aligned}
\nabla^{2} \mu_{i j \pm} & =\mp \frac{U_{00}}{D_{ \pm}} F_{00 r}(r) \frac{d \psi_{e q i j}}{d r} \cos \theta, \\
\nabla^{2} \psi_{i j}-\kappa^{2} \psi_{i j} & =-\frac{\kappa^{2}}{2}\left(\mu_{i j+}-\mu_{i j-}\right) .
\end{aligned}
$$

The boundary conditions for $\mathbf{u}_{i j}, \mu_{i j \pm}$, and $\psi_{i j}$ are

$$
r=r_{0}: \mathbf{u}_{i j}=\mathbf{0}
$$




$$
\begin{gathered}
\frac{\partial \mu_{i j \pm}}{\partial r}=0, \\
\frac{\partial \psi_{i j}}{\partial r}=0
\end{gathered}
$$

$r=a: \mathbf{u}_{i j}, \mu_{i j \pm}$, and $\psi_{i j}$ are continuous,

$\left(\mathbf{n} \cdot \boldsymbol{\sigma}_{i j}^{H}\right), \frac{\partial \mu_{i j \pm}}{\partial r}$, and $\frac{\partial \psi_{i j}}{\partial r}$ are continuous;

$r \rightarrow \infty: \mathbf{u}_{i j} \rightarrow-U_{i j} \mathbf{e}_{z}$,

$\mu_{i j \pm}$ and $\psi_{i j}$ are zero.

The solutions for the $r$ and $\theta$ components of $\mathbf{u}_{i j}, p_{i j}, \mu_{i j \pm}$, and $\psi_{i j}$ subject to Eqs. [A9] - [A15] are

$$
\begin{aligned}
u_{i j r} & =U_{i j} F_{00 r}(r) \cos \theta, \\
u_{i j \theta} & =U_{i j} F_{00 \theta}(r) \sin \theta, \\
p_{i j} & =\frac{\eta}{a} U_{i j} F_{P 00}(r) \cos \theta, \\
\mu_{i j \pm} & =U_{00} F_{i j \pm}(r) \cos \theta, \\
\psi_{i j} & =U_{00} F_{\psi i j}(r) \cos \theta,
\end{aligned}
$$

where $F_{00 r}(r), F_{00 \theta}(r)$, and $F_{P 00}(r)$ are given by Eq. [A4], and

$$
\begin{aligned}
F_{i j \pm}(r)= & \pm \frac{1}{3 D_{ \pm}}\left[\frac{r_{0}^{3}}{2 r^{2}} \int_{r_{0}}^{\infty} F_{00 r}(r) \frac{d \psi_{e q i j}}{d r} d r\right. \\
& +r \int_{r}^{\infty} F_{00 r}(r) \frac{d \psi_{e q i j}}{d r} d r \\
& \left.+\frac{1}{r^{2}} \int_{r_{0}}^{r} r^{3} F_{00 r}(r) \frac{d \psi_{e q i j}}{d r} d r\right], \quad[\mathrm{A} 1 \\
F_{\psi i j}(r)= & \frac{1}{2 \kappa r^{2}}\left\{\alpha _ { 1 } ( \kappa r ) \int _ { r } ^ { \infty } ( 1 + \kappa r ) e ^ { - \kappa r } \left[F_{i j+}(r)\right.\right. \\
& \left.-F_{i j-}(r)\right] d r+\frac{\alpha_{2}\left(\kappa r_{0}\right)-\alpha_{1}\left(\kappa r_{0}\right)}{\left[\left(\kappa r_{0}\right)^{2}+2 \kappa r_{0}+2\right] e^{-\kappa r_{0}}} \\
& \times(\kappa r+1) e^{-\kappa r} \int_{r_{0}}^{\infty}(1+\kappa r) e^{-\kappa r}\left[F_{i j+}(r)\right. \\
& \left.-F_{i j-}(r)\right] d r+(\kappa r+1) e^{-\kappa r} \int_{r_{0}}^{r} \alpha_{1}(\kappa r) \\
& \left.\times\left[F_{i j+}(r)-F_{i j-}(r)\right] d r\right\} .
\end{aligned}
$$

In Eqs. [A19] and [A20], the definite integrals can be performed numerically. Note that $F_{i j \pm}$ and $F_{\psi i j}$ take the same forms both in the regions $r_{0}<r<a$ and $r>a$.

\section{Second-Order Perturbations}

Among the second-order terms in the perturbation procedure, the only distributions we need in the calculations in Sections 4 and 5 for the sedimentation velocity and potential are the velocity $\mathbf{u}_{i j}$ and pressure $p_{i j}$ with $(i, j)$ equal to $(0$, $2),(1,1)$, and $(2,0)$. The equations governing $\mathbf{u}_{i j}$ and $p_{i j}$ are

$$
\begin{gathered}
\nabla^{2} \mathbf{u}_{02}-h(r) \lambda^{2} \mathbf{u}_{02}=\frac{1}{\eta} \nabla p_{02}-\frac{\epsilon}{4 \pi \eta} \\
\times\left(\nabla^{2} \psi_{e q 01} \nabla \psi_{01}+\nabla^{2} \psi_{01} \nabla \psi_{e q 01}\right), \\
\nabla \cdot \mathbf{u}_{02}=0
\end{gathered}
$$

$$
\begin{gathered}
\nabla^{2} \mathbf{u}_{11}-h(r) \lambda^{2} \mathbf{u}_{11}=\frac{1}{\eta} \nabla p_{11}-h(r) \frac{\epsilon \kappa^{2} k T}{4 \pi \eta z e} \nabla \psi_{01} \\
-\frac{\epsilon}{4 \pi \eta}\left(\nabla^{2} \psi_{e q 01} \nabla \psi_{10}+\nabla^{2} \psi_{e q 10} \nabla \psi_{01}\right. \\
\left.+\nabla^{2} \psi_{01} \nabla \psi_{e q 10}+\nabla^{2} \psi_{10} \nabla \psi_{e q 01}\right) \\
\nabla \cdot \mathbf{u}_{11}=0
\end{gathered}
$$

$\nabla^{2} \mathbf{u}_{20}-h(r) \lambda^{2} \mathbf{u}_{20}=\frac{1}{\eta} \nabla p_{20}-\frac{\epsilon}{4 \pi \eta}\left(\nabla^{2} \psi_{e q 10} \nabla \psi_{10}\right.$

$$
\begin{gathered}
\left.+\nabla^{2} \psi_{10} \nabla \psi_{e q 10}\right)-h(r) \frac{\epsilon \kappa^{2} k T}{4 \pi \eta z e} \nabla \psi_{10}, \\
\nabla \cdot \mathbf{u}_{20}=0 .
\end{gathered}
$$

The boundary conditions for $\mathbf{u}_{i j}$ are also given by Eq. [A2] with the subscript 00 being replaced by $i j$.

The solutions for the second-order perturbations of the components of $\mathbf{u}_{i j}$ and $p_{i j}$ are

$$
\begin{aligned}
u_{i j r}= & {\left[U_{i j} F_{00 r}(r)+U_{00} F_{i j r}(r)\right] \cos \theta, } \\
u_{i j \theta}= & {\left[U_{i j} F_{00 \theta}(r)+U_{00} F_{i j \theta}(r)\right] \sin \theta, } \\
p_{02}= & {\left[\frac{\eta}{a} U_{02} F_{P 00}(r)+\frac{\eta}{a} U_{00} F_{P 02}(r)\right.} \\
& \left.+U_{00} \frac{\epsilon \kappa^{2}}{4 \pi} \psi_{e q 01}(r) F_{\psi 01}(r)\right] \cos \theta, \\
p_{11}= & \left\{\frac{\eta}{a} U_{11} F_{P 00}(r)+\frac{\eta}{a} U_{00} F_{P 11}(r)\right. \\
& +U_{00} \frac{\epsilon \kappa^{2}}{4 \pi}\left[\psi_{e q 01}(r) F_{\psi 10}(r)\right. \\
& \left.\left.+\psi_{e q 10}(r) F_{\psi 01}(r)\right]\right\} \cos \theta,
\end{aligned}
$$




$$
\begin{aligned}
p_{20}= & {\left[\frac{\eta}{a} U_{20} F_{P 00}(r)+\frac{\eta}{a} U_{00} F_{P 20}(r)\right.} \\
& \left.+U_{00} \frac{\epsilon \kappa^{2}}{4 \pi} \psi_{e q 10}(r) F_{\psi 10}(r)\right] \cos \theta
\end{aligned}
$$

where

$$
\begin{aligned}
F_{i j r}(r)= & C_{i j 1}+C_{i j 2}\left(\frac{a}{r}\right)^{3}+C_{i j 3}\left(\frac{a}{r}\right)^{3} \alpha_{1}(\lambda r) \\
& +C_{i j 4}\left(\frac{a}{r}\right)^{3} \beta_{1}(\lambda r)+\frac{2}{(\lambda a)^{2}}\left[\int_{a}^{r} G_{i j}(r) d r\right. \\
& \left.-\left(\frac{a}{r}\right)^{3} \int_{a}^{r}\left(\frac{r}{a}\right)^{3} G_{i j}(r) d r\right] \\
& +\frac{6 \alpha_{1}(\lambda r)}{(\lambda a)^{2}(\lambda r)^{3}} \int_{a}^{r} \beta_{1}(\lambda r) G_{i j}(r) d r \\
& -\frac{6 \beta_{1}(\lambda r)}{(\lambda a)^{2}(\lambda r)^{3}} \int_{a}^{r} \alpha_{1}(\lambda r) G_{i j}(r) d r \\
F_{i j \theta}(r)= & -C_{i j 1}+\frac{C_{i j 2}}{2}\left(\frac{a}{r}\right)^{3}-\frac{C_{i j 3}}{2}\left(\frac{a}{r}\right)^{3} \alpha_{2}(\lambda r) \\
& -\frac{C_{i j 4}}{2}\left(\frac{a}{r}\right)^{3} \beta_{2}(\lambda r)-\frac{2}{(\lambda a)^{2}} \int_{a}^{r} G_{i j}(r) d r \\
& -\frac{a}{\lambda^{2} r^{3}} \int_{a}^{r}\left(\frac{r}{a}\right)^{3} G_{i j}(r) d r-\frac{3 \alpha_{2}(\lambda r)}{(\lambda a)^{2}(\lambda r)^{3}} \\
& \times \int_{a}^{r} \beta_{1}(\lambda r) G_{i j}(r) d r \\
& \frac{3 \beta_{2}(\lambda r)}{(\lambda a)^{2}(\lambda r)^{3}} \int_{a}^{r} \alpha_{1}(\lambda r) G_{i j}(r) d r \\
&
\end{aligned}
$$$$
F_{P i j}(r)=\lambda^{2} a r\left[-C_{i j 1}+\frac{C_{i j 2}}{2}\left(\frac{a}{r}\right)^{3}\right]
$$$$
-2 \frac{r}{a} \int_{a}^{r} G_{i j}(r) d r-\left(\frac{a}{r}\right)^{2}
$$$$
\times \int_{a}^{r}\left(\frac{r}{a}\right)^{3} G_{i j}(r) d r, \quad \text { if } r_{0}<r<a ;
$$

$$
\begin{aligned}
F_{i j r}(r)= & C_{i j 5}\left(\frac{a}{r}\right)^{3}+C_{i j 6} \frac{a}{r}+C_{i j 7}+C_{i j 8}\left(\frac{r}{a}\right)^{2} \\
& +\frac{1}{5}\left(\frac{a}{r}\right)^{3} \int_{a}^{r}\left(\frac{r}{a}\right)^{5} G_{i j}(r) d r-\frac{a}{r} \int_{a}^{r}\left(\frac{r}{a}\right)^{3} G_{i j}(r) d r \\
& +\int_{a}^{r}\left(\frac{r}{a}\right)^{2} G_{i j}(r) d r-\frac{1}{5}\left(\frac{r}{a}\right)^{2} \int_{a}^{r} G_{i j}(r) d r
\end{aligned}
$$

[A26d]

[A26a]

[A26c]

$$
\begin{aligned}
F_{i j \theta}(r)= & \frac{C_{i j 5}}{2}\left(\frac{a}{r}\right)^{3}-\frac{C_{i j 6}}{2}\left(\frac{a}{r}\right)-C_{i j 7}-2 C_{i j 8}\left(\frac{r}{a}\right)^{2} \\
& +\frac{1}{10}\left(\frac{a}{r}\right)^{3} \int_{a}^{r}\left(\frac{r}{a}\right)^{5} G_{i j}(r) d r+\frac{a}{2 r} \int_{a}^{r}\left(\frac{r}{a}\right)^{3} \\
& \times G_{i j}(r) d r-\int_{a}^{r}\left(\frac{r}{a}\right)^{2} G_{i j}(r) d r \\
& +\frac{2}{5}\left(\frac{r}{a}\right)^{2} \int_{a}^{r} G_{i j}(r) d r, \\
F_{P i j}(r)= & C_{i j 6}\left(\frac{a}{r}\right)^{2}+10 C_{i j 8} \frac{r}{a}-2 \frac{r}{a} \int_{a}^{r} G_{i j}(r) d r \\
& -\left(\frac{a}{r}\right)^{2} \int_{a}^{r}\left(\frac{r}{a}\right)^{3} G_{i j}(r) d r, \quad \text { if } a<r .
\end{aligned}
$$

In Eq. [A26], functions $G_{i j}(r), \alpha_{1}(x), \alpha_{2}(x), \beta_{1}(x)$, and $\beta_{2}(x)$ are defined by Eqs. [41] and [A5];

$$
\begin{aligned}
C_{i j 1}= & -\frac{\lambda^{2} a^{3}}{3 r_{0}}\left[C_{i j 3} \sinh \left(\lambda r_{0}\right)+C_{i j 4} \cosh \left(\lambda r_{0}\right)\right] \\
& -\frac{2}{(\lambda a)^{2}} \int_{a}^{r_{0}} G_{i j}(r) d r+\frac{2}{\lambda^{3} a^{2} r_{0}}\left[\cosh \left(\lambda r_{0}\right)\right. \\
& \times \int_{a}^{r_{0}} \alpha_{1}(\lambda r) G_{i j}(r) d r-\sinh \left(\lambda r_{0}\right) \\
& \left.\times \int_{a}^{r_{0}} \beta_{1}(\lambda r) G_{i j}(r) d r\right],
\end{aligned}
$$

$C_{i j 2}=2 C_{i j 1}+\frac{2}{(\lambda a)^{2}}\left(C_{i j 6}+10 C_{i j 8}\right)$,

$$
C_{i j 3}=\frac{3}{(\lambda a)^{3} \Omega}\left\{\left[A+3 \lambda r_{0}\left(\beta_{1}(\lambda a)-\beta_{1}\left(\lambda r_{0}\right)\right)\right] C_{i j}\right.
$$$$
+5\left[A+6 \lambda a \cosh \left(\lambda r_{0}\right)-3 \lambda r_{0}(\lambda a\right.
$$$$
\left.\left.\times \sinh (\lambda a)+\cosh (\lambda a)+\beta_{1}\left(\lambda r_{0}\right)\right)\right] C_{i j 8}
$$
$+\frac{2 r_{0}}{\lambda a^{2}}\left[2(\lambda a)^{3} \sinh (\lambda a)+\left(\lambda r_{0}\right)^{2}\right.$

$\left.\times \cosh \left(\lambda r_{0}\right)+3 \beta_{1}(\lambda a)-3 \beta_{1}\left(\lambda r_{0}\right)\right] \int_{a}^{r_{0}} G_{i j}(r) d r$ $-\frac{2}{(\lambda a)^{2}}\left[B \cosh \left(\lambda r_{0}\right)-3\left(\lambda r_{0}\right)^{2} \sinh (\lambda a) \sinh \left(\lambda r_{0}\right)\right]$ $\times \int_{a}^{r_{0}} \alpha_{1}(\lambda r) G_{i j}(r) d r+\frac{2}{(\lambda a)^{2}}\left[B \sinh \left(\lambda r_{0}\right)\right.$ $\left.-3\left(\lambda r_{0}\right)^{2} \sinh (\lambda a) \cosh \left(\lambda r_{0}\right)+3 \lambda r_{0}\right]$ 


$$
\begin{aligned}
& \times \int_{a}^{r_{0}} \beta_{1}(\lambda r) G_{i j}(r) d r+2 \lambda a \Delta \\
& \left.\times \int_{a}^{r_{0}}\left(\frac{r}{a}\right)^{3} G_{i j}(r) d r\right\},
\end{aligned}
$$

$$
C_{i j 4}=\frac{1}{(\lambda a)^{3} \Delta}\left\{3 \lambda r_{0} C_{i j 7}+15 \lambda r_{0} C_{i j 8}-(\lambda a)^{3}\right.
$$

$$
\begin{aligned}
& \times\left[\lambda r_{0} \cosh (\lambda a)-\sinh \left(\lambda r_{0}\right)\right] C_{i j 3} \\
& +\frac{6}{(\lambda a)^{2}}\left[\sinh \left(\lambda r_{0}\right) \int_{a}^{r_{0}} \beta_{1}(\lambda r) G_{i j}(r) d r\right.
\end{aligned}
$$$$
\left.-\cosh \left(\lambda r_{0}\right) \int_{a}^{r_{0}} \alpha_{1}(\lambda r) G_{i j}(r) d r\right]
$$

$$
\left.+\frac{6 r_{0}}{\lambda a^{2}} \int_{a}^{r_{0}} G_{i j}(r) d r\right\}
$$

$$
\begin{aligned}
C_{i j 5}= & C_{i j 1}+C_{i j 2}+\alpha_{1}(\lambda a) C_{i j 3}+\beta_{1}(\lambda a) \\
& \times C_{i j 4}-C_{i j 6}-C_{i j 7}-C_{i j 8} \\
C_{i j 6}= & C_{005} \int_{a}^{\infty} G_{i j}(r) d r+C_{006} \int_{a}^{\infty}\left(\frac{r}{a}\right)^{2} G_{i j}(r) d r
\end{aligned}
$$$$
-C_{001} \int_{a}^{r_{0}}\left(\frac{r}{a}\right)^{3} G_{i j}(r) d r-C_{002} \int_{a}^{r_{0}} G_{i j}(r) d r
$$$$
-C_{003} \int_{a}^{r_{0}} \alpha_{1}(\lambda r) G_{i j}(r) d r
$$$$
-C_{004} \int_{a}^{r_{0}} \beta_{1}(\lambda r) G_{i j}(r) d r,
$$

$$
C_{i j 7}=-\int_{a}^{\infty}\left(\frac{r}{a}\right)^{2} G_{i j}(r) d r
$$

$$
C_{i j 8}=\frac{1}{5} \int_{a}^{\infty} G_{i j}(r) d r
$$

where $C_{00 k}$ (with $k=1,2, \ldots, 6$ ), $\Delta, \Omega$, and $A$ are defined by Eqs. [A6], [A7a], [A7b], and [A8], respectively, and

$$
\begin{aligned}
B= & {\left[2(\lambda a)^{3}+\left(\lambda r_{0}\right)^{3}+3 \lambda a+3 \lambda r_{0}\right] } \\
& \times \sinh (\lambda a)-3 \cosh (\lambda a) .
\end{aligned}
$$

[A28]

The definite integrals in Eqs. [A26] and [A27] can be performed numerically.

\section{ACKNOWLEDGMENT}

This research was supported by the National Science Council of the Republic of China under Grant NSC86-2214-E-002-013.

\section{REFERENCES}

1. Booth, F., J. Chem. Phys. 22, 1956 (1954).

2. Saville, D. A., Adv. Colloid Interface Sci. 16, 267 (1982).

3. Levine, S., Neale, G., and Epstein, N., J. Colloid Interface Sci. 57, 424 (1976)

4. Stigter, D., J. Phys. Chem. 84, 2758 (1980).

5. Wiersema, P. H., Leob, A. L., and Overbeek, J. Th. G., J. Colloid Interface Sci. 22, 78 (1966).

6. de Groot, S. R., Mazur, P., and Overbeek, J. Th. G., J. Chem. Phys. 20, 1825 (1952).

7. Ohshima, H., Healy, T. W., White, L. R., and O'Brien, R. W., J. Chem. Soc., Faraday Trans. 2 80, 1299 (1984).

8. Stigter, D., J. Phys. Chem. 86, 3553 (1982).

9. Chen, S. B., and Koch, D. L., J. Colloid Interface Sci. 180, 466 (1996).

10. Napper, D. H., "Polymeric Stabilization of Colloidal Dispersions." Academic Press, London, 1983.

11. Anderson, J. L., and Solomentsev, Y., Chem. Eng. Commun. 148-150, 291 (1996).

12. Wunderlich, R. W., J. Colloid Interface Sci. 88, 385 (1982).

13. Sasaki, S., Colloid Polymer Sci. 263, 935 (1985).

14. Masliyah, J. H., Neale, G., Malysa, K., and van de Ven, T. G. M., Chem. Eng. Sci. 42, 245 (1987).

15. Prasad, D., Narayan, K. A., and Chhabra, R. P., Int. J. Eng. Sci. 28, 215 (1990).

16. Jones, I. S., J. Colloid Interface Sci. 68, 451 (1979).

17. Levine, S., Levine, M., Sharp, K. A., and Brooks, D. E., Biophys. J. 42, 127 (1983).

18. Sharp, K. A., and Brooks, D. E., Biophys. J. 47, 563 (1985).

19. Ohshima, H., Nakamura, M., and Kondo, T., Colloid Polym. Sci. 270, 873 (1992).

20. Hsu, J. P., and Fan, Y. P., J. Colloid Interface Sci. 172, 230 (1995).

21. Kawahata, S., Ohshima, H., Muramatsu, N., and Kondo, T., J. Colloid Interface Sci. 138, 182 (1990).

22. Morita, K., Muramatsu, N., Ohshima, H., and Kondo, T., J. Colloid Interface Sci. 147, 457 (1991).

23. Makino, K., Yamamoto, S., Fujimoto, K., Kawaguchi, H., and Ohshima, H., J. Colloid Interface Sci. 166, 251 (1994).

24. Ohshima, H., J. Colloid Interface Sci. 163, 474 (1994).

25. O'Brien, R. W., J. Colloid Interface Sci. 81, 234 (1981).

26. Blaakmeer, J., Bohmer, M. R., Cohen Stuart, M. A., and Fleer, G. J., Macromolecules 23, 2301 (1990).

27. Liu, Y. C., and Keh, H. J., Colloids Surf. A. [In press]

28. Hermans, J. J., and Fujita, H., Proc. Akad. Amsterdam B 58, 182 (1955).

29. van de Ven, T. G. M., "Colloidal Hydrodynamics." Academic Press, London, 1989.

30. Anderson, J. L., J. Colloid Interface Sci. 105, 45 (1985).

31. Solomentsev, Y. E., Pawar, Y., and Anderson, J. L., J. Colloid Interface Sci. 158, 1 (1993).

32. Keh, H. J., and Liu, Y. C., J. Colloid Interface Sci. 172, 222 (1995).

33. Anderson, J. L., and Kim, J. O., J. Chem. Phys. 86, 5163 (1987). 